
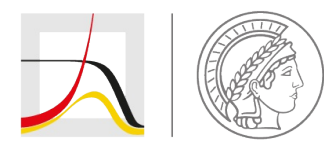

MAX PLANCK INSTITUTE

FOR DEMOGRAPHIC RESEARCH

Konrad-Zuse-Strasse 1 · D-18057 Rostock · Germany · Tel +49 (0) 3812081 - 0 · Fax +49 (0) 3812081 - $202 \cdot$ www.demogr.mpg.de

MPIDR Working Paper WP 2020-034 I October 2020

https://doi.org/10.4054/MPIDR-WP-2020-034

\title{
COVID-19 and the Future of US Fertility: What Can We Learn from Google?
}

\author{
Joshua Wilde I wilde@demogr.mpg.de \\ Wei Chen I chen@demogr.mpg.de \\ Sophie Lohmann I lohmann@demogr.mpg.de
}

This working paper has been approved for release by: Natalie Nitsche (nitsche@demogr.mpg.de),

Deputy Head of the Laboratory of Fertility and Well-Being.

(c) Copyright is held by the authors.

Working papers of the Max Planck Institute for Demographic Research receive only limited review. Views or opinions expressed in working papers are attributable to the authors and do not necessarily reflect those of the Institute. 


\title{
COVID-19 and the Future of US Fertility:
}

\section{What Can We Learn from Google?}

\author{
Joshua Wilde \\ Wei Chen \\ Sophie Lohmann *
}

October 6, 2020

\begin{abstract}
We use data from Google Trends to predict the effect of the COVID-19 pandemic on future births in the United States. First, we show that periods of above-normal search volume for Google keywords relating to conception and pregnancy in US states are associated with higher numbers of births in the following months. Excess searches for unemployment keywords have the opposite effect. Second, by employing simple statistical learning techniques, we demonstrate that including information on keyword search volumes in prediction models significantly improves forecast accuracy over a number of cross-validation criteria. Third, we use data on Google searches during the COVID-19 pandemic to predict changes in aggregate fertility rates in the United States at the state level through February 2021. Our analysis suggests that between November 2020 and February 2021, monthly US births will drop sharply by approximately $15 \%$. For context, this would be a $50 \%$ larger decline than that following the Great Recession of 2008-2009, and similar in magnitude to the declines following the Spanish Flu pandemic of 1918-1919 and the Great Depression. Finally, we find heterogeneous effects of the COVID-19 pandemic across different types of mothers. Women with less than a college education, as well as Black or African American women, are predicted to have larger declines in fertility due to COVID-19. This finding is consistent with elevated caseloads of COVID-19 in low-income and minority neighborhoods, as well as with evidence suggesting larger economic impacts of the crisis among such households.
\end{abstract}

Keywords: COVID-19, Google, Fertility, Prediction, Statistical Learning JEL codes: J11, J13, I10, C53.

*Joshua Wilde: Corresponding Author. Max Planck Institute for Demographic Research. Konrad-Zuse-Straße 1, 18057 Rostock, Germany. E-mail: wilde@demogr.mpg.de. Wei Chen: Department of Economics, Fordham University, The Bronx, NY 10458. E-mail: chen@demogr.mpg.de. Sophie Lohmann: Max Planck Institute for Demographic Research. Konrad-Zuse-Straße 1, 18057 Rostock, Germany. E-mail: lohmann@ demogr.mpg.de. This research was improved by discussions with (in alphabetical order) Padmaja Ayyagari, Kieron Barclay, Kasey Buckles, Jennifer Caputo, Audrey Dorelien, Peter Eibich, Willa Friedman, Kathryn Grace, Melanie Guldi, Mahesh Karra, Mine Kühn, Giulia La Mattina, Susie Lee, Mikko Myrskyla, Daniela Negraia, Natalie Nitsche, Anna Oksuzyan, Lucie Schmidt, Alyson van Raalte, David Weil, and Emilio Zagheni. Author Contributions according to the CREDIT taxonomy: Joshua Wilde: Conceptualization, Methodology, Formal Analysis, Writing - Original Draft; Wei Chen: Investigation, Data Curation, Writing - Review \& Editing; Sophie Lohmann: Software, Data Curation, Writing - Review \& Editing. The authors declare no competing interest. 


\section{Introduction}

The COVID-19 pandemic has had significant consequences for human mortality. At their peak, all-age weekly excess deaths exceeded $14 \%$ in Germany, $45 \%$ in the US, $90 \%$ in Italy, and $154 \%$ in Spain[1]. While most attention is focused on the current mortality and economic consequences of the pandemic, its effects on fertility are currently unknown[2]. In spite of this uncertainty, some in the popular media have suggested the pandemic will result in a "baby boom" as couples spend more time together. Such pronouncements are viewed with skepticism by many demographers, citing evidence on the short-term fertility effects from other mortality crises, including natural disasters [3] [4] [5] [6] and previous pandemics [7] [8]. These studies generally show that mortality spikes are followed by reductions in births within a year, with some evidence of fertility rebounds after several years [9]. Theoretically, these declines are explained by couples' aversion to childbearing during periods of economic uncertainty, in an unhealthy environment, or by increased spontaneous pregnancy termination due to stress or disease [10]. While some rebounds have been even larger than the initial decline and led to long-run increases in fertility, these usually occur when mortality spikes are concentrated in children as parents attempt to replace deceased offspring — a mechanism which should be less relevant for the COVID-19 pandemic, since it primarily affects mortality among older individuals [5] [6].

Fertility change has significant economic and social consequences[11] [12]. Reductions in birthrates accelerate population ageing, increasing dependency ratios in populations already far below replacement fertility, such as in Southern Europe and East Asia[13]. This can lower economic growth by reducing the fraction of working age population, while simultaneously increasing the burden of caring for the elderly, both through public social programs as well as private channels[14] [15]. However, these social and economic effects are mainly affected by long-run fertility change. If the mortality crises only leads to a postponement of childbearing and leaves lifetime births per woman unchanged, the long-run economic effects from postponement should be minimal. However, recent postcrisis fertility declines - including the 2008-2009 financial crisis - did not experience a rebound and led to permanently lower fertility rates. Since the exact nature of the effect of COVID-19 on the future of human fertility is unclear, the economic and social affects of the crisis due to demographic change is also unknown.

Human gestation takes on average 268 days, so there is a natural delay from the onset of these crises and their effect on full-term births [16]. For example, full-term births from conceptions realized during the rapid onset of the pandemic in February or March of 2020 would not appear until November or December. Unfortunately, this delay in understanding the effect of the pandemic on fertility is compounded since natality data generally does not become available for analysis instantaneously. For example, the US Natality File birth microdata from the National Center for Health Statistics (NCHS) are generally released at least 6-9 months after the end of the year in which those births occurred. Therefore, the earliest analyses of the pandemic's beginnings on birth outcomes using these traditional data will not be available until late 2021, with more detailed analyses including births from 
early 2021 not becoming available until late 2022. Even in countries which have faster data releases, significant delays between the advent of births and the release of data hamper the ability of researchers to make timely analyses of the relationship between COVID-19 and fertility rates.

An emerging literature suggests that data on Google searches can be used to monitor a number of social and biological phenomena in the absence of more reliable or timely data. Such data have been used for real-time analyses of disease outbreaks such as the seasonal flu and Dengue[17] [18], as well as studies on well-being [19], tourism [20], financial trading behavior [21], and demographic processes such as fertility [22], migration [23], sexual behavior [24] [25] [26], and mortality [27]. Moving beyond now-casting with Google data is generally difficult due to complexity or uncertainty surrounding the longterm processes which govern such phenomena. Fortunately, behavior and informationseeking surrounding human gestation takes place in predictable phases, and with wellknown time lags, making Google search data particularly appealing for prediction. As an example, morning sickness generally only occurs during the first trimester, so an increase in searches for morning sickness and its symptoms may help pinpoint an increase in the fertility rate over half a year in the future, and with an accuracy of just a few months.

In addition to uncertainty surrounding the pandemic's effect on fertility, there may be important heterogeneous effects across sub-national regions, or between socioeconomic groups. For example, COVID-19 incidence and mortality have been elevated among the Black or African American community in the US, and the economic impacts have been particularly acute for workers with lower levels of education[28] [29]. Additionally, while planned births may fall as the economic fallout of the pandemic induces couples to delay childbearing, reduced contraceptive access may lead to an increase in unintended pregnancies. This effect is particularly acute for areas with historically poor contraceptive access: A 2020 UNFPA report noted that COVID-19 is already excacerbating unmet family planning needs due to a variety of reasons, including decreased demand for health facility visits, unavailability of trained medical staff, and supply chain distruptions for contraceptive commodities[30]. Analyzing differential changes in Google search volumes across regions with varying proportions of ethnic and other socioeconomic groups may yield deeper insights into the potential effects of COVID-19 on fertility.

In addition, heterogeneous changes in the types of keywords searched across regions may help pinpoint specific mechanisms by which the pandemic will affect fertility. For example, information-seeking on Google regarding emergency contraception may indicate possible changes in unplanned pregnancies, while searches for miscarriages may indicate fetal loss. Finally, inasmuch as behavior regarding conception and sexual behavior is considered by many to be socially taboo, some individuals may be more willing to search for such information on the internet than to discuss their behavior in person [26]. Therefore, although Google data is only an imperfect reflection of the behaviors which affect fertility, it may more accurately reflect actual behavioral change than a direct self-report of those behaviors. 
In this study, we use high-frequency data Google search term volume for keywords related to fertility to predict the direction, magnitude, and timing of fertility change expected from the pandemic in the United States at the state level. We do this in four steps. First, we show that periods of above-normal search volume for Google keywords relating to conception and pregnancy are associated with higher numbers of births in the following months. Excess searches for unemployment keywords has the opposite effect. Second, by employing simple statistical learning techniques, we demonstrate that including information on keyword search volumes in prediction models significantly improves forecast accuracy over a number of cross-validation criteria. Third, we use data on Google searches during the COVID-19 pandemic to predict changes in aggregate fertility rates in the United States at the state level through February 2021. Finally, we test for heterogeneous effects of the COVID-19 pandemic on fertility by education, ethnic identity, and age of mother, and by parity of birth.

\section{Data and Methods}

\section{A Google Data}

Our data on keyword search frequency comes from Google Trends (http://trends.google.com), a website which allows users to access data on Google keyword search frequency, stratified by geographic regions ranging from as large as a country to as small as a city. Data for smaller geographic areas can be more difficult to use, since the data are suppressed unless the overall search volume reaches a minimum threshold. For this article, we use data from the state level in the United States to ensure a wide selection of available search terms, yet still preserve geographic variation in search frequency.

\section{B Natality Data}

Data on births by state and month are from the National Vital Statistics System (NVSS) which is part of the National Center for Health Statistics (NCHS). Monthly birth counts for each US state and the District of Columbia from 2004-2018 were used, since 2004 was the year the Google search data began, and 2018 is the most recent year of data on births is available. This yields 15 years of usable data across 51 geographic regions, or 9,180 possible state-month-year observations.

\section{Methods}

\section{A Keyword Selection}

Keyword sets were created in a multi-step process. Initially, keywords were chosen by brainstorming possible words which we believed to be predictive, specific, and common 
enough for use in forecasting. We then sought input from other researchers to inform ourselves of any important topics or keywords we might be missing. Due to the necessarily arbitrary initial keyword set, all keywords were pre-selected before looking at Google data in order to avoid data-mining. Details on each selected keyword or topic and their meaning are discussed in the Supplemental Information. The list of inital keywords or topics are: Baby Heartbeat, BabyCenter, Birth Control, Clearblue, Conceive, Cytotec, Dilation and Curettage, Divorce, Emergency Contraception, File Unemployment How, Folate, Furlough, Human chorionic gonadotropin (hCG), Intra-uterine Device (IUD), In-vitro Fertilization (IVF), Layoff, Medical Abortion, Midwife, Miscarriage, Misoprostol, Missed Period, Morning After Pill, Morning Sickness, Obygn, Online Dating, Ovulation, Ovulation Test, Plan B, Porn, Pregnancy, Pregnancy Bleeding, Pregnancy Symptoms, Pregnancy Test, Pregnant, Prenatal Vitamins, Sexually Transmitted Infection (STI), Ultrasound, Unemployment, Unemployment Office, Unprotected Sex, and a control set. We then subjected each keyword to a series of three screens in order to test goodness of fit and predictive power. These screens are mentioned in the main text, and described more fully later in this section and in the Supplemental Information. In addition, details on our statistical learning method for selecting the most predictive keywords for the MSPE Reduction keyword set are also outlined in the Supplemental Information.

\section{B Prediction Model}

Our baseline prediction model is an OLS regression as follows:

$$
Y_{s m y}=\alpha_{s m}+\sum_{s=1}^{2} \gamma_{s} * t^{s}+\sum_{w}^{W} \sum_{l=t_{0}}^{T} \beta_{w, t-l} I_{s m y}^{w}+\epsilon_{s m y}
$$

where $s, m$, and $y$ index state, month, and year respectively, $\alpha_{s m}$ is a state-month fixed effect, $\gamma_{s} * t$ is a state specific linear and quadratic time trend. The independent variables $I_{s m y}^{w}$ are the natural $\log$ of the normalized search volume as given by the google data and whose construction are outlined in the supplemental information. The double summation represents a series of $\beta$ coefficients for the effects of different keywords search volumes at a number of monthly time lags. The dependent variable $Y$ is the natural log of births, implying the interpretation of the $\beta$ s are an elasticity — the percentage change in births from each percentage change in monthly search volume. We use $t_{0}$ of 7 and a $T$ of 12, representing monthly time lags from 7-12 months before birth. Huber-White standard errors are utilized for estimation.

\section{C Data Screens and Cross-Validation Techniques}

We use three data screens to reduce our initial keyword set. We first omits any keyword for which more than $1 / 3$ of the 9,180 state-month observations were missing. Second, we further omitted any keywords that were not jointly or individually significant predictors of births for the lagged months 7-12. We call this the Significance screen, which selected 
14 keywords for inclusion, listed in alphabetical order: ClearBlue, Divorce, HCG, IVF, Layoff, Morning Sickness, Ovulation, Porn, Pregnancy Test, Pregnancy, Pregnant, STI, Ultrasound, and Unemployment.

Next, the MSPE Reduction screen used a statistical learning procedure called forward step-wise selection to select the keywords from the Significance screen which lead to the largest reduction in mean squared prediction error (MPSE). To find this MSPE reduction, we used a variation of the "Leave-One-Out" cross-validation technique in which for each tested keyword set, we omit two years of data, train the data using the remaining 13 years, and then use the estimated associations to predict births in the omitted two years. We then calculate the prediction error as the absolute value - in percentage terms - of the difference between these predictions and the actual births for every state-month. We then repeat for every possible combination of two years of data, resulting in 105 model runs. We then average the mean squared prediction error across the runs to find the MSPE for that keyword set.

The forward step-wise selection learning procedure proceeds as follows. First, the base model without any keywords is estimated. Then, we add each keyword one at a time to the model (and a control keyword) and employ the MSPE calculation procedure described above for each word. The word which minimizes the MSPE the most is selected, and becomes part of the base model. Then a second round begins, where each remaining word is selected to be added to this new base model one at a time, and the word which minimizes the MSPE is selected. This procedure continues until the additional reduction is MSPE is less than one percentage point. This screen selected five keywords, listed in order of selection: Unemployment, HCG, Clearblue, Unemployment Office, and Ovulation. (See Supplemental Information for more details.)

Additionally, two extra topical screens were utilized to explore heterogeneity between word types as described in the article — the Early indicators screen (including the words Clearblue, HCG, Morning Sickness, Ovulation, Pregnancy Test, Pregnancy, Pregnant), and Unemployment screen (including the words Unemployment, Unemployment Office, and Layoff).

\section{Results}

In our first step, we tested associations between 40 fertility-related keywords and monthly births at the state level in the United States between 2004 and 2018. Our keywords are listed in the Material and Methods section, with further descriptions and keyword selection methodologies are found in the Supplemental Information. Our estimation methodology utilized an Ordinary Least Squares (OLS) regression which controlled for seasonality in both births and keyword searches, which controls for time-independent state effects and state-specific time polynomials. We also included a set of control keywords as a proxy for overall internet use.

We estimated the month-specific effect of each keyword on births for each month be- 
tween 1 to 12 months before the observed state-level births, and plot these results for a subset of keywords in Figure 1. Specifically, we show the results for seven of the most predictive keywords (ClearBlue, Morning Sickness, IVF, Ovulation, Pregnancy, Ultrasound, and Unemployment) and our control keyword set. These findings confirm that there are intuitive associations between fertility-related keywords and future births. For example, excess searches for "ClearBlue" - the name of a popular brand of pregnancy test in the US - is significantly associated with more births 5-8 months later, with the largest effect 7 and 8 months later. This is consistent with the timing when many pregnant women are experiencing their first pregnancy symptoms at 4-8 weeks after conception. Similarly, excess searches for "Morning Sickness" are associated with more births 6-8 months later, corresponding with the first trimester when morning sickness generally occurs.

For our second step, we conduct model cross-validation to show that including subsets of pregnancy related keywords in our estimation model reliably improve the predictive power beyond our baseline model. This is important, since simple associations between individual keywords may be spurious, or a result of model over-fitting. We use wellaccepted statistical learning methodologies and divide our data into a training data set and a test data set - the first of which is used to estimate the associations between keywords and births, and the second to test the predictive power of the keywords out-of-sample. We find that including these keywords increases forecast model precision by approximately $25 \%$ over a number of criteria. Descriptions of these cross-validation techniques and their results are given in the Supplemental Information section.

Third, we used these estimated associations between future births and pregnancyrelated keyword searches to forecast state-level births through February 2021 using Google search volumes up to July 2020. These results are displayed in Figure 2, in which statespecific predictions using our model with Google searches are aggregated to the national level, and then shown relative to the same predictions using the model without searches. Displaying our results in terms of this relative index has the benefit of showing the predicted deviation in births between the models in percentage terms relative to what would be otherwise expected in a comparable month. We compared four different keyword sets against this baseline, where the selection criteria for each are fully explained in the Supplemental Information. Briefly however, the Significance keyword set contains those keywords which were both jointly significant across all monthly lags included in our model and individually significant for at least one monthly lag; the MSPE keyword set was the set of words which minimized the Mean Square Prediction Error (MSPE) using a forward step-wise keyword selection criteria; the Early keyword set only included words which were statistically significant and concerned early pregnancy keywords unrelated to health services; an Unemployment keyword set which only contained statistically significant keywords related to unemployment.

Each of the keyword sets - with the exception of Early pregnancy indicators - show similarly striking results: beginning in November 2020, fertility is predicted to fall by several percentage points each month until February 2021. For example, using the MSPE re- 
duction keyword set, we predict the number of births in February 2021 will be just $84.3 \%$ of what they would have been for a normal February. Estimates for the Unemployment keyword set are almost identical to those for the MSPE Reduction keyword set. The Significance keyword set predicts a smaller - yet still very large — decline to just $88.1 \%$ of expected births. All of these declines are highly statistically significant at the $0.1 \%$ level. However, the Early indicators keyword set shows almost no decline at all - fertility is predicted to be a statistically insignificant $98.8 \%$ of its normal level.

These disparate estimates can be explained by examining changes in Google search volume for specific keywords as a result of the pandemic. In the Supplemental Information, we show Google keyword search volume for the keywords shown in Figure 1, after having been corrected for seasonality. Several patterns emerge in these figures. First, whereas searches for many words fell dramatically around the first state-wide shutdown, many fertility-related search terms have remained surprisingly stable throughout the crisis. The words which did decline have a unifying commonality: they all relate to prenatal or conception services which must be experienced in a clinical setting. For example, searches related to "Obgyn", "Ultrasound", and "IVF" (In-vitro fertilization) all fell sharply. However, it is unlikely that searches for ultrasounds - which generally are associated in our data with pregnancies in the late 1st and 2nd trimester - fell sharply due to significant declines in conceptions in December and January. The abrupt decline in searches for these words likely reflected an interruption in clinical services due to state-wide shutdowns and social distancing behavior. Conversely, highly predictive words which do not involve prenatal services such as "ClearBlue", "Morning Sickness", "Ovulation", and "Pregnancy" were essentially unaffected by the coronavirus pandemic. Therefore, in spite of their predictive power, forecasts based solely on these keywords - such as our Early indicators keyword set - imply no large negative effect of the pandemic on fertility.

However, the results are reversed for any keyword set which includes words related to unemployment. It is well documented that fertility is sensitive to economic conditions, and generally follows a counter-cyclical pattern[10]. As seen in Figure 1, excess searches for unemployment around and immediately before the time of conception (8-11 months before birth) are strongly associated with lower birthrates. This is presumably due to some couples timing their births to avoid periods of financial hardship or economic uncertainty[10]. In addition, searches for unemployment jumped to 20 times their normal level by April - a month after the first statewide lockdown - and stabilized by the beginning of June to approximately half this amount. The combination of large changes in search volumes for unemployment and large coefficient values from our prediction model for this keyword imply that the pandemic and its subsequent economic fallout will strongly decrease fertility, as shown in the Unemployment, Significance, and MSPE keyword set estimates in Figure 2.

Finally, we explore whether our predicted effects vary across different states, or by type of birth. Among the latter, we consider four sources of heterogeneity - maternal education, age, and ethnicity, and by parity of birth. We find sizable differences across 
US states: the state with the highest predicted decline between October 2020 and February 2021 using the MSPE reduction set is Hawaii with reduction of $23.6 \%$, while the lowest decline is found in Connecticut of only $11.9 \%$. As shown in the Supplemental Information, the largest predicted declines are generally in the Southern US and the Rust Belt, with the exception of Hawaii. In Figure 3, we only present our results based on differences in maternal education and ethnicity - those for parity and maternal age are found in the Supplemental Information, and demonstrate somewhat smaller declines for first births and no differences by maternal age.

In Figure 3, we find that mothers with less of education or who identify as Black or African American are predicted to have sharper declines in births. We do this in two ways. First, we change the dependent variable in our prediction model from all births in a state to only births for each educational or ethnic subgroup, and present the same relative birth index as in Figure 2, but now broken out by subgroup. For comparison, we do this analysis using the Significance keyword set, which predicted an average decline in fertility of $11.9 \%$ These results are found in the top row of Figure 3. Second, in the bottom row of Figure 3 we plot the overall predicted decline, by state, between October 2020 and February 2021 - after controlling for COVID-19 cases per capita and population density — against the fraction of the population in each state belonging to various ethnic or educational groups. We find that for those who have completed a Bachelor's degree or higher, the COVID-19 pandemic is predicted to have very little effect on fertility. Specifically, by Feb 2021 we only predict a statistically insignificant $3.1 \%$ decrease in fertility for this group. However, for both women with only some college, and those with only a high school degree, we predict a $13 \%$ decline. Similarly, states with a higher fraction of women with at least a Bachelor's degree have smaller predicted fertility decline than states with lower Bachelor's degree attainment. By ethnic group, we find very small effects for Hispanics (2.9\% decline) relative to non-Hispanic Whites (9.2\% decline) and Black or African Americans (15.3\% decline). We also find that states with larger Black or African American populations have larger predicted declines in fertility.

\section{Discussion}

A 15\% collapse in births over a four-month period would be unprecedented in the modern United States. Therefore, it may be useful to explore the plausibility of this prediction. To do so, we consider three major crises in the US with similarities to the current pandemic: the Spanish Flu pandemic of 1918-1919, the Great Depression of 1929-1933, and the financial crisis of 2008-2009. In doing so, it is important to keep in mind that the context of the current pandemic is fundamentally different from each of these crises, and therefore these comparisons should be taken with a measure of caution. However, as the coronavirus pandemic itself is in many ways unprecedented, these three crises arguably serve as the best comparisons at our disposal.

The first crisis we consider is the 1918-1919 H1N1 influenza A pandemic — com- 
monly known as the Spanish Flu. Birth rates fell 13\% from 1918 to 1919 in the United States, with a small rebound in 1920 and 1921[8]. This decline is strikingly similar to that predicted for the current pandemic in this study. The fertility effects of the Great Depression are also very similar in magnitude to the Spanish Flu and our prediction for the COVID-19 pandemic. Between 1929 and 1933, birthrates fell by 15.2\%[31]. Finally, the financial crisis of 2008-2009 caused a smaller decline in fertility, albeit in response to a smaller economic crisis. From May 2008 to October 2010, births fell by $9.3 \%$ and failed to rebound thereafter.

The evolution of births over these three crises suggests that a $15 \%$ decline in the fertility rate in response to the COVID-19 pandemic is not unreasonable. Using the above information on changes in unemployment and changes in births, the response of predicted births to unemployment for the current pandemic is firmly between the response from the financial crisis and the Great Depression. Calculating the elasticity of births to unemployment defined as the ratio between the percentage change in births and the percentage change in unemployment - yields an elasticity of -0.050 for the current pandemic, firmly between that of the financial crisis (-0.109) and the Great Depression (-0.027). Importantly, if there is a rebound in births later in the year, the decline in fertility could be significantly less that $15 \%$, lowering this elasticity substantially. Therefore, the main difference between the prediction of this study and the evolution of fertility during these three crises is not the magnitude of the decline, but rather the speed. Since the current pandemic has led to historically fast increases in unemployment, this suggests that similarly rapid decreases in fertility may be expected.

Our finding that individuals identifying as Black or African American, or with lower educational attainment, have larger predicted declines in fertility can be interpreted in two ways. First, areas with high concentrations of African Americans have been more impacted by COVID-19: incidence among Black individuals was 2.6 times higher than for non-Hispanic whites, and mortality was 2.1 times higher[28]. Since individuals of lower socioeconomic status are disproportionately affected by the virus, this could manifest in higher predicted fertility declines[29]. Second, it may be that the heterogeneity in the predicted fertility effect is not caused by the differential impact of the virus itself, but rather from the uneven economic fallout of the pandemic. Historically, during recessions employment losses are often concentrated among minority groups - and among Black individuals in particular - and the current economic downturn is no exception. Between Feburary and April of 2020, the unemployment rate among non-Hispanic White Americans rose from $3.1 \%$ to $14.2 \%$, while for Black or African Americans it rose from 5.8\% to $16.7 \%$. While these initial increases were similar, the recovery has been much slower for Black or African Americans: by August of 2020 non-Hispanic White unemployment had fallen back to $7.3 \%$, yet Black unemployment remained elevated at $13.0 \%$. The patterns in unemployment between educated and uneducated workers were even more striking, peaking at only $8.4 \%$ for those with a Bachelor's degree or higher, compared with $17.3 \%$ and $21.2 \%$ for non-college graduates with and without a high school diploma respectively[32]. 
There are several important caveats to this study which should be noted. First, the evidence presented here can be interpreted in two ways. According to the first interpretation, since google search volume related to pregnancy behavior and symptoms alone - and the corresponding fertility prediction - did not change significantly over the course of the pandemic, there is little reason to think that there will be a large decrease in births as a result. In this view, the effect of the large implied changes in unemployment can be ignored, because if high unemployment searches truly implied fewer future births, we would expect fewer searches for morning sickness, pregnancy tests, and other fertility keywords as a result. We do not observe such reductions. However, the second interpretation concludes that because unemployment searches are strongly associated with future births, and because these searches have spiked since the beginning of the pandemic, fertility will decline by approximately $15 \%$. Since our methodology shows that searches related to unemployment are far more predictive than the rest of our keywords combined, an objective data science perspective would favor this interpretation. Importantly however, neither interpretation includes the possibility of a large increase in births, notwithstanding recent speculation.

The second important caveat is that this method can only predict fertility change up to 9-12 months in the future, leaving the long-run effects of the pandemic unknown. If the decline in fertility is simply due to a postponement in births, completed lifetime fertility may remain relatively unchanged. However, even if these predicted declines are primarily due to couples postponing children they still intend to have, this may still negatively affect long-run fertility. Since the 1970s there has been a shift towards births at later ages as women postpone childbearing for educational or career motives[33]. Since fecundity declines rapidly towards the end of a woman's childbearing years, women in their late $30 \mathrm{~s}$ and early 40s who further delay childbearing due to the pandemic may face unexpected difficulties trying to conceive, leading to unintentionally lower completed lifetime fertility [34]. If this happens, the resulting lower completed fertility and accompanying shift to an older population age structure has significant social and economic consequences, particularly for regions already well below replacement fertility. Decreasing tax revenue, greater costs for social programs for the elderly, pension liabilities, increased health care costs, reduced economic growth, and increased burdens of caring for the elderly are all important policy issues surrounding falling fertility rates and the resulting aging of the population. [33] [15] [11] [12]. 


\section{References}

[1] Centers for Disease Control. Excess deaths associated with covid-19, 2020.

[2] A. Aassve, N. Cavalli, L. Mencarini, S. Plach, and M. Livi Bacci. The COVID-19 pandemic and human fertility. Science, 369(6502):370-371, July 2020.

[3] Jocelyn E. Finlay. Fertility Response To Natural Disasters: The Case Of Three High Mortality Earthquakes. POLICY RESEARCH WORKING PAPERS, apr 2009.

[4] Richard W. Evans, Yingyao Hu, and Zhong Zhao. The fertility effect of catastrophe: U.S. hurricane births. Journal of Population Economics, 23(1):1-36, nov 2009.

[5] Jenna Nobles, Elizabeth Frankenberg, and Duncan Thomas. The Effects of Mortality on Fertility: Population Dynamics After a Natural Disaster. Demography, 52(1):1538, jan 2015.

[6] Julia Andrea Behrman and Abigail Weitzman. Effects of the 2010 Haiti Earthquake on Women's Reproductive Health. Studies in Family Planning, 47(1):3-17, mar 2016.

[7] Jacques Bertillon. Annuaire statistique de la ville de Paris. Imprimerie nationale, 1895.

[8] Siddharth Chandra, Julia Christensen, Svenn-Erik Mamelund, and Nigel Paneth. Short-term birth sequelae of the 1918-1920 influenza pandemic in the United States: state-level analysis. American Journal of Epidemiology, 187(12):2585-2595, 2018.

[9] Alberto Palloni. On the Role of Crises in Historical Perspective: An Exchange: Comment. Population and Development Review, 14(1):145-158, 1988.

[10] Tomáš Sobotka, Vegard Skirbekk, and Dimiter Philipov. Economic recession and fertility in the developed world, jun 2011.

[11] Quamrul H. Ashraf, David N. Weil, and Joshua Wilde. The effect of fertility reduction on economic growth. Population and Development Review, 39(1):97-130, mar 2013.

[12] Mahesh Karra, David Canning, and Joshua Wilde. The Effect of Fertility Decline on Economic Growth in Africa: A Macrosimulation Model. Population and Development Review, 43:237-263, may 2017.

[13] Population Reference Bureau. 2020 world population data sheet, 2020.

[14] Nicole Maestas, Kathleen J. Mullen, and David Powell. The effect of population aging on economic growth, the labor force and productivity. NBER Working Paper No. 22452, 2016.

[15] John R. Beard and David E. Bloom. Towards a comprehensive public health response to population ageing. The Lancet, 385(9968):658-661, February 2015.

[16] A.M. Jukic, D.D. Baird, C.R. Weinberg, D.R. McConnaughey, and A.J. Wilcox. Length of human pregnancy and contributors to its natural variation. Human Reproduction (Oxford, England), 28(10):2848-2855, October 2013. 
[17] Jeremy Ginsberg, Matthew Mohebbi, Rajan Patel, Lynnette Brammer, Mark Smolinski, and Larry Brilliant. Detecting influenza epidemics using search engine query data. Nature, 457:1012-1014, 2009.

[18] Herman Anthony Carneiro and Eleftherios Mylonakis. Google trends: a web-based tool for real-time surveillance of disease outbreaks. Clinical Infectious Diseases: An Official Publication of the Infectious Diseases Society of America, 49(10):15571564, November 2009.

[19] Abel Brodeur, Andrew E Clark, Sarah Flèche, and Nattavudh Powdthavee. COVID19, Lockdowns and Well-Being: Evidence from Google Trends. page 55, 2020.

[20] Boriss Siliverstovs and Daniel S. Wochner. Google Trends and reality: Do the proportions match? Journal of Economic Behavior \& Organization, 145(C):1-23, 2018.

[21] Tobias Preis, Helen Susannah Moat, and H. Eugene Stanley. Quantifying Trading Behavior in Financial Markets Using Google Trends. Scientific Reports, 3(1):1684, April 2013.

[22] Francesco Billari, Francesco D’Amuri, and Juri Marcucci. Forecasting Births Using Google. In Proceedings of the 1st International Conference on Advanced Research Methods and Analytics. Universitat Politècnica València, July 2016.

[23] Emilio Zagheni and Ingmar Weber. You are where you e-mail: using e-mail data to estimate international migration rates. In WebSci '12, 2012.

[24] Patrick M. Markey and Charlotte N. Markey. Seasonal Variation in Internet Keyword Searches: A Proxy Assessment of Sex Mating Behaviors. Archives of Sexual Behavior, 42(4):515-521, May 2013.

[25] Joshua Wilde, Bénédicte H. Apouey, and Toni Jung. The effect of ambient temperature shocks during conception and early pregnancy on later life outcomes. European Economic Review, 97:87-107, August 2017.

[26] Seth Stephens-Davidowitz. Everybody Lies: Big Data, New Data, and What the Internet Can Tell Us About Who We Really Are. Harper-Collins, 2017.

[27] J. K. Tamgno, R. M. Faye, and C. Lishou. Verbal autopsies, mobile data collection for monitoring and warning causes of deaths. 2013 15th International Conference on Advanced Communications Technology (ICACT), 2013.

[28] Centers for Disease Control. Covid-19 hospitalization and death by race/ethnicity, 2020.

[29] W. Holmes Finch and Maria E. Hernández Finch. Poverty and covid-19: Rates of incidence and deaths in the united states during the first 10 weeks of the pandemic. Frontiers in Sociology, 5:47, 2020.

[30] UNFPA - United Nations Population Fund. Impact of the COVID-19 Pandemic on Family Planning and Ending Gender-based Violence, Female Genital Mutilation and Child Marriage. Technical report, 2020. 
[31] Robert Heuser. Fertility tables for birth cohorts by color. Number DHEW Publication No. (HRA)76-1152. U.S. Department of Health, Education, and Welfare.

[32] Bureau of Labor Statistics. Unemployment rate - series lns14000003, lns14000006, lns14027662, Ins14027660, and lns14027659, 2020.

[33] GBD 2017 Population and Fertility Collaborators. Population and fertility by age and sex for 195 countries and territories, 1950-2017: a systematic analysis for the Global Burden of Disease Study 2017. The Lancet, 392(10159):1995-2051, 2018.

[34] Emmanuel Attali and Yariv Yogev. The impact of advanced maternal age on pregnancy outcome. Best Practice Research Clinical Obstetrics Gynaecology, 2020. 
Figure 1: Fertility Keyword Searches and Later Births. These figures show regression coefficients between births in a state and Google keyword search volume for the preceding 12 months. Coefficients are elasticities, and can be interpreted as the change in births due to a doubling of keyword search volume in a given month. Dashed lines represent $95 \%$ confidence intervals.
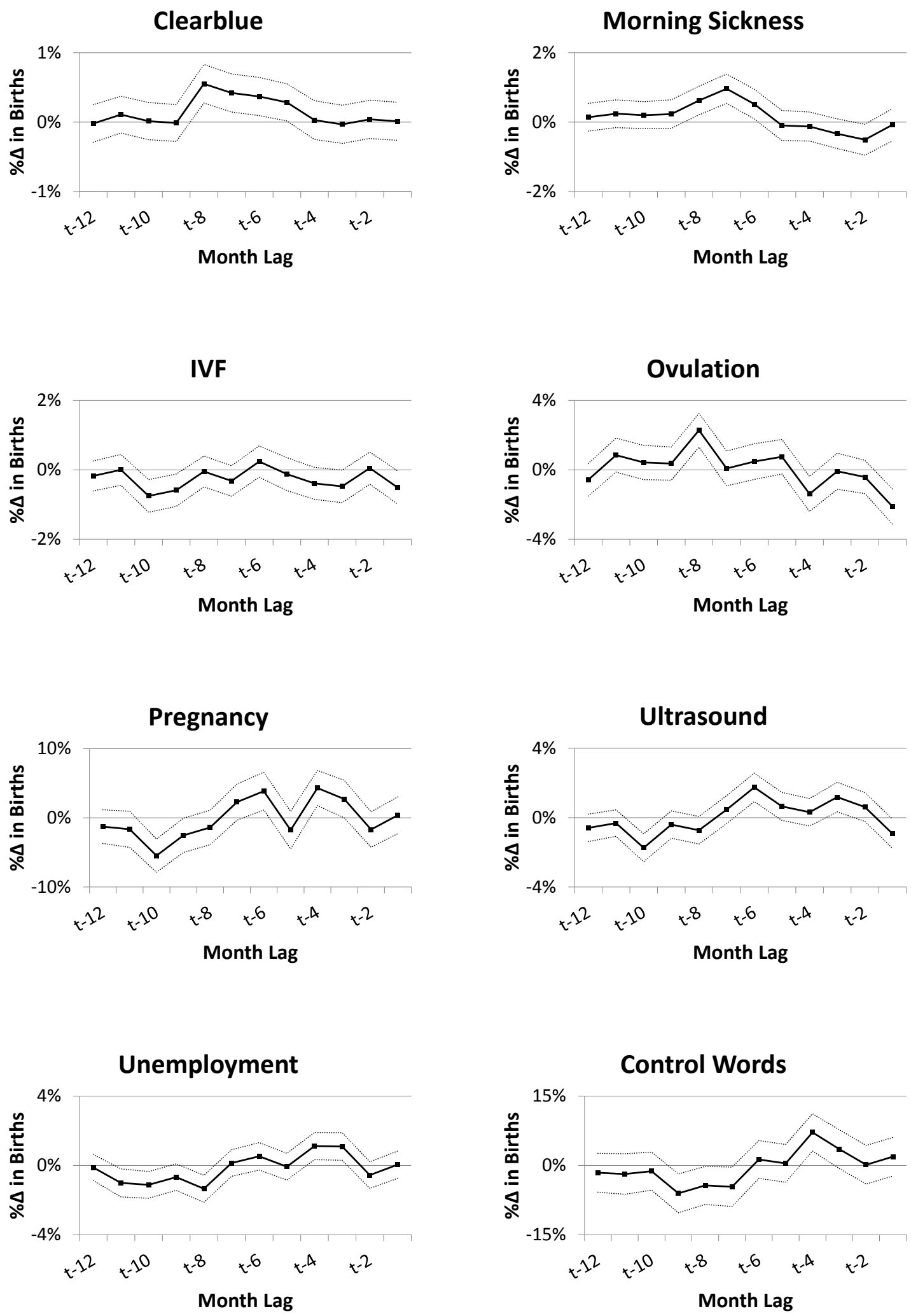
Figure 2: Predicted 2020 US Births by Month. National predicted births, for various keyword sets, relative to the corresponding prediction without keyword searches, and normalized to 100 in October 2020. The four keyword sets are: Significance, including keywords whose associations meet statistical significance thresholds; MSPE, including keywords selected from a mean-squared prediction error minimizing forward step-wise learning selection criteria; Early, including keywords related to early pregnancy; and Unemployment, including keywords related to economic conditions.

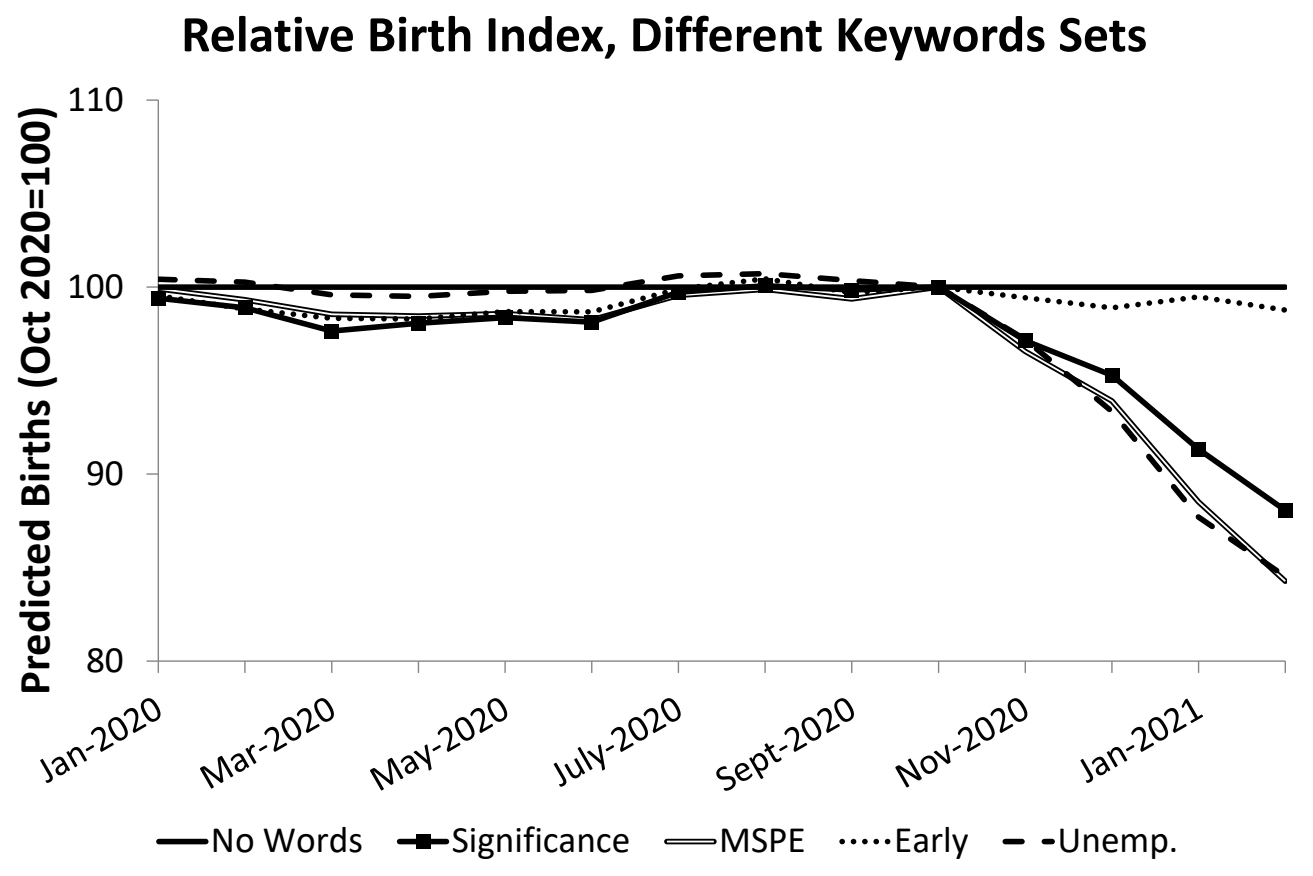


Figure 3: Predicted Fertility Decline by Various Mother Characteristics. The top panel shows national predicted births, by group, for the Significance keyword set, relative to the corresponding prediction without keyword searches, and normalized to 100 in October 2020. The bottom panel shows a scatter-plot relating the predicted fertility decline between Oct. 2020 and Feb. 2021 - after controlling for COVID-19 cases per capita and population density - by the fraction of the population in the respective subgroup in that state. Data source: American Community Survey 2013-2017, authors calculations.

\section{Education}

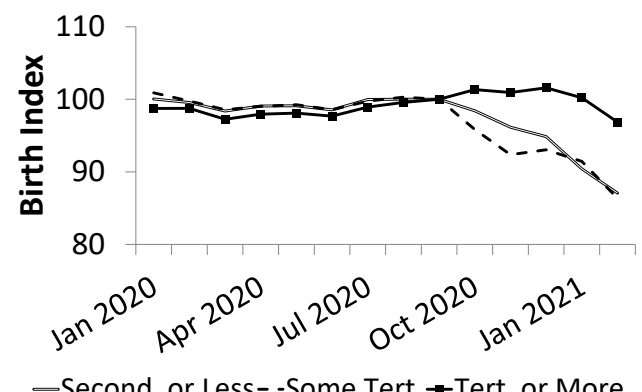

-Second. or Less- -Some Tert. - Tert. or More

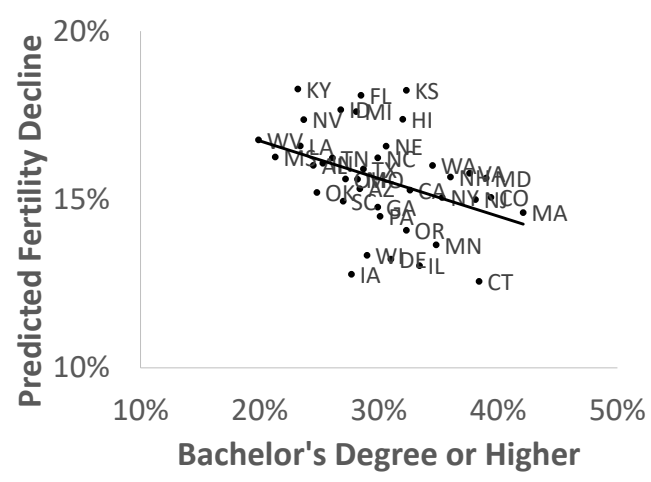

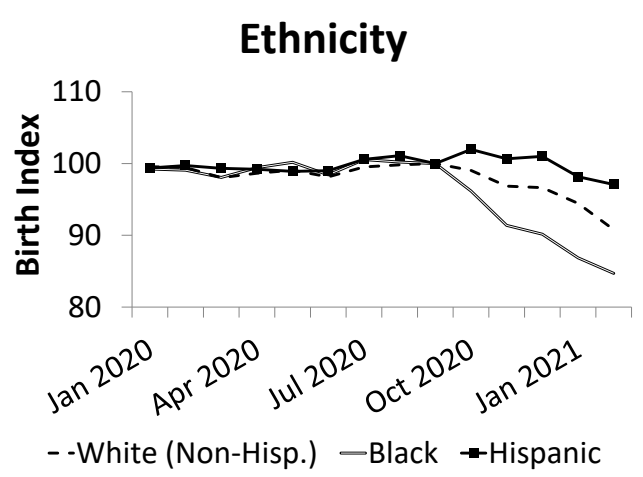

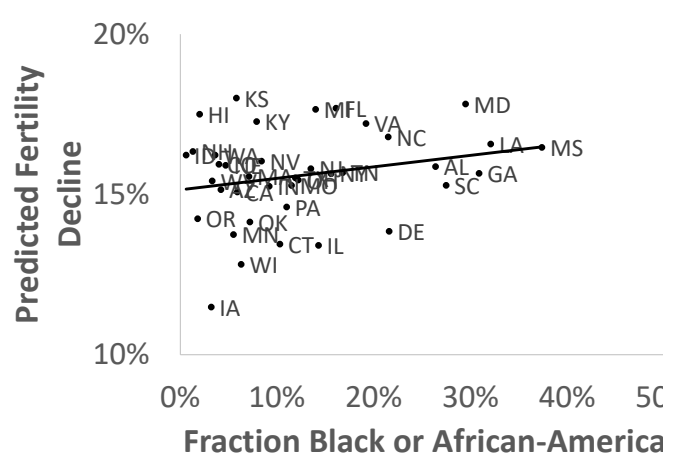




\section{Supplemental Information}

\section{Word Selection Methodology}

Choosing a set of keywords with which to analyze search frequency is not straightforward for several reasons. First, there is no a priori agreed upon set of keywords which should be both theoretically associated with - and empirically predictive of — pregnancy. Second, words associated with pregnancy generally have multiple meanings, which meanings can also change over time. ${ }^{1}$ Third, different keyword sets may have different associations for different types of pregnancy behaviors. ${ }^{2}$ Finally, the same keywords may theoretically have different correlations with births at various pregnancy timeframes. ${ }^{3}$

The lack of a defined, agreed upon pregnancy keyword set may lead to several statistical and ethical problems when determining which keywords to include. For example, an unscrupulous researcher may datamine keywords to find those which are correlated with pregnancy, ignore those which don't, and present the highly predictive nature of these keywords as evidence that Google searches are excellent at predicting births. Even consciously ethical researchers may be tempted to engage in unconscious motivated reasoning to include or exclude words once they see the results on how predictive they actually are. Unfortunately, without a predetermined word set, part of the word selection process is necessarily at least partially arbitrary.

\section{Pregnancy and Birth Keywords}

The first step in our word selection process was to consult a number of sources to identify possible words. We consulted the literature on Google keywords and any aspect of fertility - unfortunately, these are very scarce. The only paper we could find specifically on pregnancy and Google searches was Billari et al (2013), which utilized three keywords ovulation, pregnancy, and maternity. Other papers utilized keyword sets which, while related to pregnancy, were not pregnancy specific. For example, Markey and Markey (2013) developed a keyword set for pornographic, sexual, and mate-seeking keywords, which was subsequently used in Wilde, Apouey, and Jung (2017) and Wilde, Lohmann, and Chen

\footnotetext{
${ }^{1}$ For example, the term "Plan B" - a popular brand-name emergency contraceptive in the United States which can reduce unplanned pregnancies - could also refer to generic back-up plans completely unrelated to pregnancy. Another example is the word "Tinder" - before 2012, it referred to a type of fireword, yet after 2012 the majority of searches referred to a online dating app.

${ }^{2}$ For example, searches for emergency contraceptives may indicate a higher incidence of risky or unplanned sexual behavior, which may lead to more births. However, the same searches for emergency contraceptive may be an indication that for the same amount of risky sexual behavior, a higher proportion of those acts are accompanied by actions to reduce unplanned pregnancy, leading to fewer pregnancies.

${ }^{3}$ For example, searches regarding pregnancy or ovulation tests may be positively associated with pregnancy 9 to 11 months later as it suggests more individuals may be trying to get pregnant. However, a high frequency of searches for these same tests will likely be negatively associated with births 1 to 5 months later, since if a large fraction of women in a population are currently pregnant, there should be a lower fraction of women trying to get pregnant.
} 
(2020). Additionally, Lohmann and Albarracin (2019) developed an exhaustive keyword set for sexually transmitted infections. We then consulted online sources such as such as pregnancy and maternity websites, dictionaries, and thesauruses. Finally, we conducted a number of interviews with women which had experiences with different aspects of pregnancy, such as miscarriage, unexpected pregnancies, abortion, etc.

After this initial round, we reduced our keyword set to those 40 keywords which we believed would be 1) specific enough to be highly correlated with births, 2) broad enough to have enough data to run our analysis, and 3) which would sufficiently cover each of the following seven aspects of pregnancy: Unplanned Pregnancies, Pregnancy Intentions, Pregnancy Symptoms, Prenatal Services, Miscarriage, Economic Indicators, and Other. Importantly, we determined this final keyword set before looking at any data. These words and word groupings can be found in Table 1 in this supplement.

Once fixing this initial keyword set, we employed three systematic screens to allow us to objectively choose keyword subsets for analysis without conscious or subconscious bias in word selection. All three of these screens take a data-oriented approach. The first screen regarded sample size - if the search frequency for a given keyword was missing for more than $1 / 3$ of the total state-month observations, it did not pass screen one. Of our 40 initial words, only 6 did not pass this screen: Morning After Pill, Unprotected Sex, Baby Heartbeat, Cytotec, File Unemployment How, and Furlough. Screens two and three were data-driven relevance screens, are referred to as the Significance and MPSE screens in the body of the paper, and will be described in detail in the Methodology section of this supplement. In short, a word passed screen two if it was jointly partially correlated with births at specific time lags in our main regression specification, and passed screen 3 if it was selected by a commonly used variable selection algorithm.

\section{Control Keywords}

Our method for determining control keywords was similar to that which determined pregnancy keywords, in that we began by selecting a large set of possible control words. We combined a set of two keyword groups: commonly searched words as reported by Google, and common - but not specific - words in the English language. Specifically, we created an initial keyword set of the 25 most searched words reported by Google in the United States from 2004 to the present, in addition to 40 of the most commonly used words in the English language: the nominative and accusative personal pronouns (me, you, I, us, we, they, she, he, it); the major interrogative pronouns and other "w"-words (who, what, where, why, how, which, whose, whom); definite and indefinite articles (the, a, an); common conjunctions (and, but, or); superlatives (best, worst); various conjugations of common verbs (be, is, are, were, do, does, did, have, has, had, can, could, would, should); and "of". Of these 65 words, the top ten words with the highest search volumes are: the, of, how, a, is, what, and, me, you, and do. Searches for these ten words comprise over $50 \%$ of the combined search volume for the larger 65 word control set. For simplicity, we use these top ten as our control set. In practice, our control search variable is a single aggregate search 
index for any search involving any of these 10 words, standardized in the same manner as described below in the Google Data section.

\section{Data}

\section{Google Data}

Our data on keyword search frequency comes from Google Trends (http://trends.google.com), a website which allows users to access data on Google keyword search frequency, stratified by geographic regions ranging from as large as a country to as small as a city. Data for smaller geographic areas can be more difficult to use, since the data are suppressed unless the overall search volume reaches a minimum threshold. For this paper, we use data from the state level in the United States to ensure a wide selection of available search terms, yet still preserve geographic variation in search frequency.

Google Trends does not provide information on the actual number of keyword searches. Rather, it provides a relative search index which reports aggregate searches as a percentage of the most searched term and time period, which is given the value of 100. Therefore, the search index may vary depending on the time frame requested, as well as the set of keywords in a given query. In order to standardize the data, we requested the data for the entire 15-year sample one keyword and state at a time. We then divided the keyword frequency for each word by the state-specific mean, giving us a search frequency index for each state and word with a mean value of 1 . Therefore, each search frequency observation can be interpreted as the percentage of the average search frequency for a given word in a given state. For example, in the descriptive statistics in Table 2, the maximum search frequency for the term "unemployment" is reported as 3.72. This means that for that specific month and year in that state, searches were 3.72 times higher than the monthly state average for that word over the 15-year period. One benefit of specifying the search frequency in this fashion is that it is easy to interpret - coefficients in our regression will represent the effect of a doubling of search frequency from the mean on the outcome of interest. Missing, incomplete, or relatively low frequency search data are reported as 0 , which we set to missing.

Search data in Google can be specified as either a keyword or topic, which are measured differently. For keywords, Google reports searches with an exact keyword match, while for topic searches, Google utilizes an algorithm which attempts to include other searches which have to do with the keyword, but may not include the actual keyword itself. Unfortunately, this algorithm is proprietary to Google, and therefore is somewhat of a black box.

In Table 1, we report each of our search terms, whether we utilized the keyword or topic search data, and whether the keyword passed a given screen. Topical searches were prioritized over keywords when possible. In order to understand these terms, a note on the logic behind the Google Trends search algorithm is necessary. Certain delimiters, such as 
", -, and + allow users to change the combinations of keywords searched. A search for a single keyword will yield the search frequency index counting all searches that contain that keyword, including searches which contain other words. For example, the reported search volume for keyword "pregnant" will contain searches for "am I pregnant". If more than one keyword is entered, the resulting Google search volume will contain only searches which contain both words in the same search. In essence, the space delimiter serves as the Boolean logic operator "and". The Boolean "or" operator is "+". For example, the search Emergency Contraception will contain only searches which contain both "Emergency" and "Contraception", while the search Emergency+Contraception will contain all searches which either contain "Emergency" or "Contraception". The "-" operator removes searches containing a specified keyword. For example, one of our search terms mentioned in the paper was "HCG" short for Human chorionic gonadotropin, a hormone produced by a woman's body for the maternal recognition of pregnancy. Testing for the presence of this hormone in urine serves as the basis for many pregnancy tests. However, in the early 2010s, a fad diet called "The HCG Diet" rose then fell in popularity, leading to increases in searches for HCG unrelated to pregnancy. Therefore, we specified our search query as HCG - Weight - Diet, which kept searches for HCG, but removed any search which contained HCG but also included either the word Weight or Diet. Finally, keyword searches in quotations require an exact string match. Returning to our example, the search Emergency Contraception will include all searches which include both the words "Emergency" and "Contraception", independent of where those words appear in the query, whereas the search "Emergency contraception" will only include searches which that exact string. Therefore, a search for "Where do I find contraception in an emergency" would be counted in the former search, but not the latter.

Returning to Table 1, the keywords are listed exactly how they were queried by the system, including the ", -, and + operators. There are two exceptions. The first is the Control Set, which as explained in the Control Keywords section, was specified as the + of + how $+\mathrm{a}+\mathrm{is}+\mathrm{what}+\mathrm{and}+\mathrm{me}+$ you + do. The other exception is Online Dating. For this query, we found that both the keyword and the topic yielded unbelievably low search volume given the popularity of online dating. We concluded that a better query would be a composite term including combined searches for popular dating sites. Therefore, for our online dating keyword, we used the search term match.com + tinder + okcupid + bumble + zoosk + eHarmony + POF + plenty of fish, reflecting a combination of the seven most popular online dating platforms used over our sample period (Note that POF is merely an abbreviation for the site Plenty of Fish, whose url is pof.com).

\section{Natality Data}

Data on births by state and month are from the National Vital Statistics System (NVSS) which is part of the National Center for Health Statistics (NCHS). From this system, we get monthly birth counts for each US state and the District of Columbia. We only utilize births in years since 2004 as this is the year Google search data begins. As of this writing, the 
most recent year of data on births is 2018 , giving us a total of 15 years of monthly birth data at the state level, or 9,180 state-month-year observations. In addition, this system allows users to aggregate births by various sub-groupings, such as educational attainment, age of mother, etc. We used these data by these sub-groupings in our heterogeneity analysis.

\section{Methodology}

\section{Model}

Our prediction model relies on linear OLS fixed effects regression. Specifically, we estimate the following prediction model:

$$
Y_{s m y}=\alpha_{s m}+\sum_{s=1}^{2} \gamma_{s} * t^{s}+\sum_{w}^{W} \sum_{l=t_{0}}^{T} \beta_{w, t-l} I_{s m y}^{w}+\epsilon_{s m y}
$$

where $s, m$, and $y$ index state, month, and year respectively, $\alpha_{s m}$ is a state-month fixed

effect, $\sum_{s=1}^{2} \gamma_{s} * t^{s}$ is a state specific time polynomial, and the double summation represents a series of $\beta$ coefficients for the natural $\log$ of the normalized search volume for different keywords at a number of monthly time lags. The dependent variable $Y$ is the natural $\log$ of births, implying the interpretation of the $\beta$ s are an elasticity - the percentage change in births from each percentage change in search volume. We use $t_{0}$ of 7 and a $T$ of 12, representing monthly time lags from 7-12 months before birth. Huber-White standard errors are utilized. Given the state-month fixed effects, this regression effectively controls for state-specific seasonality in both births and keyword search volumes. In essence, it estimates the effect on births of larger than normal search volume for a given month, in a given state, compared with that same month and state across years. It also controls for changes in aggregate births over time specific to the state due to the linear and quadratic time terms.

For the results on the association between births and google searches at different time lags shown in Figure 1 of the main text, we expand the lagged month parameters from between 7 and 12 months before birth to 1 and 12 months in order to capture more time effects for illustrative purposes. However, since our prediction model is about early prediction, including all this information would only allow us to forecast births one month in advance. As a result, in our main prediction model we only include information on google searches 7 months before birth and earlier.

\section{Assessing Predictive Power}

The significant associations between certain keywords and later births presented in Table 1 in the main text do not necessarily imply that these keywords can effectively be used in prediction. For example, over-fitting is a common and well-known problem in forecasting. Conversely, however, if important explanatory variables are excluded, then even 
highly predictive variables may not be sufficient to accurately forecast. Therefore, there is generally a trade-off between increasing the number of explanatory variables - thereby increasing the information used in prediction — and over-fitting the data.

To explore a range of keyword sets on this trade-off spectrum, we use a set of keyword screens with varying levels of stringency. Our first, most basic screen is to omit any keyword which has more than $1 / 3$ of the 9,180 state-month observations missing. Our next screen, the Significance screen, takes the first screen and omits any keyword for which both of the following conditions are not true: 1) the month-specific estimated associations are jointly significant at the 5\% level for the lagged months 7-12 while also controlling for all other included keywords, and 2) there is at least one month among the lagged months 7-12 which is individually significant at the 5\% level. We implement this screen in an iterative process, where all Screen 1 variables are initially included in the regression equation. Keywords are removed if they fail to meet either conditions above, and then the model is re-run with the reduced keyword set and the conditions reapplied until the only words remaining are those for which both conditions are met. We report in Table 3 the results of each of four rounds which were necessary to select the final keyword set according to this methodology, and report which words were dropped at each round. This screen selected 14 keywords for inclusion, listed in alphabetical order: ClearBlue, Divorce, HCG, IVF, Layoff, Morning Sickness, Ovulation, Porn, Pregnancy Test, Pregnancy, Pregnant, STI, Ultrasound, and Unemployment.

For our third screen, we employ a statistical learning methodology which uses out-ofsample tests to determine predictive power, in order to select a keyword set which optimizes predictive power while minimizing the number of keywords. In this methodology, our data set is divided into two groups: a training data set, and a test data set. The training data set is used to estimate the associations between the words and the births, while the test data set is used to test the predictive power of the word estimates, as evaluated by changes in goodness-of-fit measures compared with a model which does not include any keywords as predictors. In the interest of computational feasibility, we use a variation on the leave-p-out cross validation methodology which omits two years of observations at a time, which we call leave-2-years-out (L2YO) cross validation. As we have 15 years of data, this creates 105 possible two-year combinations, and hence 105 model runs to test the predictive power of each keyword set.

We apply this L2YO methodology to select an optimal keyword set from among the Screen 1 keywords, using a process of forward step-wise selection, which is carries out as follows. First, the base model without any keywords is estimated. Then, we add each keyword one at a time to the model (with the control keyword), and calculate the prediction error as the absolute value in percentage terms of the difference between these predictions and the actual births for every state-month. We then repeat for each of 105 combination of two years of data. We then find the mean squared prediction error (MSPE) across the runs for that keyword set. The keyword which minimizes the MSPE is selected, and becomes part of the base model. Then a second round begins, where each remaining word is added 
to this new base model one at a time, and the new word which minimizes the MSPE is also selected. This procedure continues until the additional reduction is MSPE is less than one percentage point. In Table 4, we report for each round the word selected and the respective cumulative reduction in MSPE. Employing this screen selects five keywords, listed in order of selection: Unemployment, HCG, Clearblue, Unemployment Office, and Ovulation.

We find that both the Significance keyword set and the MSPE keyword set significantly improve forecast accuracy. To demonstrate this, we plot a moving average of the MSPE by month for each of three keyword sets between 2004-2018 - the model without keywords, the model utilizing the Significance keyword set, and the model with the MSPE keyword set. In Figure 4 we show the average MSPE across all the training data sets by year, and in Figure 5 we do the same for the test data sets. In these figures, we see that both the Significance and MSPE keyword sets lower model prediction error relative to a model without keywords. Significantly, the efficacy of using keywords to predict fertility is especially high during crises - prediction error using both keyword sets during the 20082009 financial crisis is approximately half as large as the model without keywords. This is a comforting result for the purposes of our exercise, since we are using these keywords to predict fertility during the COVID-19 crisis.

\section{Other Results}

\section{Searches and Fertility}

In this section we show how keyword search volume changed during the COVID-19 pandemic. Specifically, we plot adjusted keyword search volumes from January 1st, 2018 to July 31st, 2020 for a series of keywords. On each plot, we include two vertical lines for the reader's reference. The first line is on January 13, 2020, which corresponds with the estimated arrival date of the first COVID-19 case to the United States. The second vertical line corresponds with March 19th, the date of the first state-wide stay-at-home order in California.

Plotting the raw search data can be misleading since keyword search volumes generally follow seasonal patterns. Therefore, we first filter the data in the following manner: First, we estimate a regression model for each keyword with search volume as the dependent variable, and month and week effects as independent variables. Second, we save the residuals from this regression and add them to the average keyword volume over this time period. Third, we normalize the volume such that February 1st, 2020 equals 100, in order to see the effect of the pandemic as a percentage increase in searches 6 weeks before the stay-at-home orders were implemented.

\section{Other Robustness and Heterogeneous Effects}

In this section, we present various robustness checks, as well as additional results which were mentioned in the main text. Figure 7 presents our main results across different model 
lag structures. In the model presented in the main text, we include information on keyword searches from 7 months before birth to 12 months before birth, allowing us to predict births 7 months into the future. In this figure, we show three models, one in which we use 7-12 month keyword lags, another in which we use 8-12 month lags, and finally a third with 9-12 month lags. The model is surprisingly robust across different lag structures. Interestingly, we find that the extended model predicts a small birth rebound beginning in April 2021.

In Figure 3 in the main article, we show heterogeneous birth predictions by educational attainment and ethnic group. In Figure 8 we provide additional predictions by parity and maternal age. We find little evidence of differences by maternal age. However, we do find that first births are predicted to be less affected by the pandemic than higher parity births.

In Figure 9 we show differences in predicted fertility decline across US states. We plot a heat map by state, showing the predicted decline in fertility between October 2020 and February 2021.

In Figure 10 we show a state-level scatter plot of the predicted fertility decline between Oct 2020 and Feb 2021, versus cumulative Covid-19 cases per capita as of Jul 2020. While one might expect that areas with higher caseloads would have larger predicted declines in fertility, we find the opposite. However, this is likely explained by our findings on education - the states with the highest COVID-19 incidence were large, urban states, which also have higher levels of education than rural states.

\section{Estimated Coefficients}

Table 5 reports the estimated coefficients from our model with which we predict fertility. The top panel presents the coefficients for the MSPE keyword set, which the bottom panel presents coefficients for the Significance keyword set. 
Table 1: Keyword Selection Criteria.

\begin{tabular}{|c|c|c|c|c|}
\hline \multirow[b]{2}{*}{ Word } & \multirow{2}{*}{$\begin{array}{l}\text { Topic or } \\
\text { Keyword }\end{array}$} & \multicolumn{3}{|c|}{ Screen } \\
\hline & & 1 & 2 & 3 \\
\hline \multicolumn{5}{|l|}{ Controls } \\
\hline Control Set* & Keyword & $\mathrm{X}$ & $\mathrm{X}$ & $\mathrm{X}$ \\
\hline \multicolumn{5}{|l|}{ Unplanned Pregnancy } \\
\hline Emergency Contraception & Topic & $\mathrm{X}$ & & \\
\hline "Morning After Pill" & Keyword & & & \\
\hline Plan B & Keyword & $\mathrm{X}$ & & \\
\hline STI & Topic & $\mathrm{X}$ & $\mathrm{X}$ & \\
\hline "Unprotected Sex" & Keyword & & & \\
\hline Online Dating* & Keyword & $\mathrm{X}$ & & \\
\hline \multicolumn{5}{|l|}{ Pregnancy Intention } \\
\hline Clearblue & Keyword & $\mathrm{X}$ & $\mathrm{X}$ & 3 \\
\hline Conceive & Keyword & $\mathrm{X}$ & & \\
\hline HCG - Weight - Diet & Keyword & $\mathrm{X}$ & $\mathrm{X}$ & 2 \\
\hline IVF & Topic & $\mathrm{X}$ & $\mathrm{X}$ & \\
\hline Ovulation & Topic & $\mathrm{X}$ & $\mathrm{X}$ & 5 \\
\hline Ovulation Test & Keyword & $\mathrm{X}$ & & \\
\hline Pregnancy Test & Topic & $\mathrm{X}$ & $\mathrm{X}$ & \\
\hline \multicolumn{5}{|l|}{ Pregnancy Symptoms } \\
\hline Missed Period & Keyword & $\mathrm{X}$ & & \\
\hline Morning Sickness & Topic & $\mathrm{X}$ & $\mathrm{X}$ & \\
\hline Pregnancy Symptoms & Keyword & $\mathrm{X}$ & & \\
\hline \multicolumn{5}{|l|}{ Prenatal Services } \\
\hline BabyCenter & Topic & $\mathrm{X}$ & & \\
\hline Folate & Topic & $\mathrm{X}$ & & \\
\hline Midwife & Topic & $\mathrm{X}$ & & \\
\hline Obygn & Keyword & $\mathrm{X}$ & & \\
\hline Prenatal Vitamins & Topic & $\mathrm{X}$ & & \\
\hline Ultrasound & Keyword & $\mathrm{X}$ & $X$ & \\
\hline \multicolumn{5}{|l|}{ Pregnancy Termination } \\
\hline Baby Heartbeat & Keyword & & & \\
\hline Cytotec & Keyword & & & \\
\hline Dilation and Curettage & Topic & $\mathrm{X}$ & & \\
\hline Medical Abortion & Topic & $\mathrm{X}$ & & \\
\hline Miscarriage & Topic & $X$ & & \\
\hline Misoprostol & Topic & $\mathrm{X}$ & & \\
\hline Pregnancy Bleeding & Keyword & $\mathrm{X}$ & & \\
\hline \multicolumn{5}{|l|}{ Unemployment } \\
\hline File Unemployment How & Keyword & & & \\
\hline Furlough & Keyword & & & \\
\hline Layoff & Topic & $\mathrm{X}$ & $\mathrm{X}$ & \\
\hline Unemployment & Keyword & $\mathrm{X}$ & $\mathrm{X}$ & 1 \\
\hline "Unemployment Office" & Keyword & $\mathrm{X}$ & & 4 \\
\hline \multicolumn{5}{|l|}{ Other } \\
\hline Birth Control & Topic & $\mathrm{X}$ & & \\
\hline Divorce & Topic & $\mathrm{X}$ & $\mathrm{X}$ & \\
\hline IUD & Topic & $\mathrm{X}$ & & \\
\hline Porn & Keyword & $\mathrm{X}$ & $\mathrm{X}$ & \\
\hline Pregnancy & Topic & $\mathrm{X}$ & $X$ & \\
\hline Pregnant & Keyword & $\mathrm{X}$ & $\mathrm{X}$ & \\
\hline
\end{tabular}


Table 2: Descriptive Statistics

\begin{tabular}{|c|c|c|c|c|c|}
\hline Variable & Obs & Mean & S.d. & Min & $\operatorname{Max}$ \\
\hline \multicolumn{6}{|l|}{ Natality Variables } \\
\hline Births & 9,180 & 6,602 & 7,776 & 359 & 51,747 \\
\hline Ln(Births) & 9,180 & 8.27 & 1.04 & 5.94 & 10.85 \\
\hline \multicolumn{6}{|l|}{ Keyword/Topics } \\
\hline Control Set* & 9,180 & 1 & 0.16 & 0.64 & 1.35 \\
\hline BabyCenter & 8,744 & 1 & 0.62 & 0.07 & 8.22 \\
\hline Birth Control & 9,153 & 1 & 0.24 & 0.24 & 3.88 \\
\hline Clearblue & 7,097 & 1 & 0.71 & 0.15 & 9.03 \\
\hline Conceive & 7,916 & 1 & 0.60 & 0.17 & 12.95 \\
\hline Divorce & 9,173 & 1 & 0.22 & 0.21 & 4.64 \\
\hline Dilation \& Curettage & 7,917 & 1 & 0.65 & 0.17 & 11.84 \\
\hline Emergency Contra. & 8,725 & 1 & 0.43 & 0.14 & 8.30 \\
\hline Folate & 8,633 & 1 & 0.54 & 0.17 & 7.90 \\
\hline $\mathrm{HCG}^{*}$ & 8,641 & 1 & 0.51 & 0.15 & 6.53 \\
\hline IUD & 8,712 & 1 & 0.38 & 0.20 & 4.83 \\
\hline IVF & 8,627 & 1 & 0.52 & 0.12 & 9.31 \\
\hline Layoff & 8,575 & 1 & 0.75 & 0.15 & 11.00 \\
\hline Medical Abortion & 7,286 & 1 & 0.61 & 0.15 & 9.60 \\
\hline Midwife & 8,498 & 1 & 0.60 & 0.14 & 7.24 \\
\hline Miscarriage & 8,927 & 1 & 0.35 & 0.23 & 6.20 \\
\hline Misoprostol & 6,693 & 1 & 0.76 & 0.18 & 10.81 \\
\hline Missed Period & 7,931 & 1 & 0.51 & 0.17 & 6.83 \\
\hline Morning Sickness & 8,424 & 1 & 0.49 & 0.20 & 7.04 \\
\hline Obgyn & 8,618 & 1 & 0.41 & 0.11 & 4.06 \\
\hline Online Dating* & 9,162 & 1 & 0.42 & 0.12 & 2.31 \\
\hline Ovulation & 9,086 & 1 & 0.26 & 0.22 & 4.02 \\
\hline Ovulation Test & 7,198 & 1 & 0.59 & 0.18 & 9.72 \\
\hline Plan B & 8,398 & 1 & 0.40 & 0.11 & 6.14 \\
\hline Porn & 9,180 & 1 & 0.35 & 0.25 & 2.29 \\
\hline Pregnancy & 9,180 & 1 & 0.18 & 0.35 & 1.96 \\
\hline Pregnancy Bleeding & 7,954 & 1 & 0.61 & 0.20 & 8.90 \\
\hline Pregnancy Symptoms & 8,945 & 1 & 0.38 & 0.19 & 6.28 \\
\hline Pregnancy Test & 8,975 & 1 & 0.32 & 0.21 & 4.80 \\
\hline Pregnant & 9,177 & 1 & 0.37 & 0.12 & 2.12 \\
\hline Prenatal Vitamins & 7,838 & 1 & 0.55 & 0.19 & 6.43 \\
\hline STI & 9,064 & 1 & 0.54 & 0.18 & 8.07 \\
\hline Ultrasound & 9,069 & 1 & 0.35 & 0.16 & 5.43 \\
\hline Unemployment Office & 8,283 & 1 & 0.61 & 0.13 & 9.09 \\
\hline Unemployment & 9,155 & 1 & 0.54 & 0.09 & 3.81 \\
\hline
\end{tabular}

*See Google Data section for extra details. 
Table 3: Significance Screen Word Selection

\begin{tabular}{|c|c|c|c|c|}
\hline Word & Round 1 & Round 2 & Round 3 & Round 4 \\
\hline Clearblue & $\mathrm{X}$ & $\mathrm{X}$ & $\mathrm{X}$ & $\mathrm{X}$ \\
\hline Divorce & $X$ & $X$ & $\mathrm{X}$ & $\mathrm{X}$ \\
\hline HCG & $X$ & $X$ & $X$ & $\mathrm{X}$ \\
\hline IVF & $\mathrm{X}$ & $\mathrm{X}$ & $\mathrm{X}$ & $\mathrm{X}$ \\
\hline Layoff & $\mathrm{X}$ & $\mathrm{X}$ & $\mathrm{X}$ & $\mathrm{X}$ \\
\hline Morning Sickness & $\mathrm{X}$ & $\mathrm{X}$ & $\mathrm{X}$ & $\mathrm{X}$ \\
\hline Ovulation & $\mathrm{X}$ & $\mathrm{X}$ & $\mathrm{X}$ & $\mathrm{X}$ \\
\hline Porn & $\mathrm{X}$ & $\mathrm{X}$ & $\mathrm{X}$ & $\mathrm{X}$ \\
\hline Pregnancy Test & $\mathrm{X}$ & $\mathrm{X}$ & $\mathrm{X}$ & $\mathrm{X}$ \\
\hline Pregnancy & $\mathrm{X}$ & $\mathrm{X}$ & $\mathrm{X}$ & $\mathrm{X}$ \\
\hline Pregnant & $\mathrm{X}$ & $\mathrm{X}$ & $\mathrm{X}$ & $\mathrm{X}$ \\
\hline STI & $\mathrm{X}$ & $\mathrm{X}$ & $\mathrm{X}$ & $\mathrm{X}$ \\
\hline Ultrasound & $X$ & $X$ & $\mathrm{X}$ & $\mathrm{X}$ \\
\hline Unemployment & $\mathrm{X}$ & $\mathrm{X}$ & $\mathrm{X}$ & $\mathrm{X}$ \\
\hline Dilation \& Curettage & $\mathrm{X}$ & $\mathrm{X}$ & $\mathrm{X}$ & \\
\hline Miscarriage & $\mathrm{X}$ & $\mathrm{X}$ & & \\
\hline Obgyn & $\mathrm{X}$ & $\mathrm{X}$ & & \\
\hline Unemployment Office & $\mathrm{X}$ & $\mathrm{X}$ & & \\
\hline BabyCenter & $\mathrm{X}$ & & & \\
\hline Birth Control & $\mathrm{X}$ & & & \\
\hline Conceive & $\mathrm{X}$ & & & \\
\hline Emergency Contra. & $X$ & & & \\
\hline Folate & $\mathrm{X}$ & & & \\
\hline IUD & $X$ & & & \\
\hline Medical Abortion & $\mathrm{X}$ & & & \\
\hline Midwife & $\mathrm{X}$ & & & \\
\hline Misoprostol & $\mathrm{X}$ & & & \\
\hline Missed Period & $\mathrm{X}$ & & & \\
\hline Online Dating & $\mathrm{X}$ & & & \\
\hline Ovulation Test & $\mathrm{X}$ & & & \\
\hline Plan B & $\mathrm{X}$ & & & \\
\hline Pregnancy Bleeding & $\mathrm{X}$ & & & \\
\hline Pregnancy Symptoms & $\mathrm{X}$ & & & \\
\hline Prenatal Vitamins & $\mathrm{X}$ & & & \\
\hline
\end{tabular}

Table 4: MSPE Screen Word Selection

\begin{tabular}{llc}
\hline Round & Word & $\begin{array}{c}\text { Cumulative } \Delta \\
\text { Mean MSPE }\end{array}$ \\
\hline & & \\
1 & Unemployment & $-20.7 \%$ \\
2 & HCG & $-23.3 \%$ \\
3 & Clearblue & $-24.6 \%$ \\
4 & Unemployment Office & $-25.7 \%$ \\
5 & Ovulation & $-26.7 \%$ \\
6 & STI & $-27.5 \%$ \\
\hline
\end{tabular}


Figure 4: Training Error

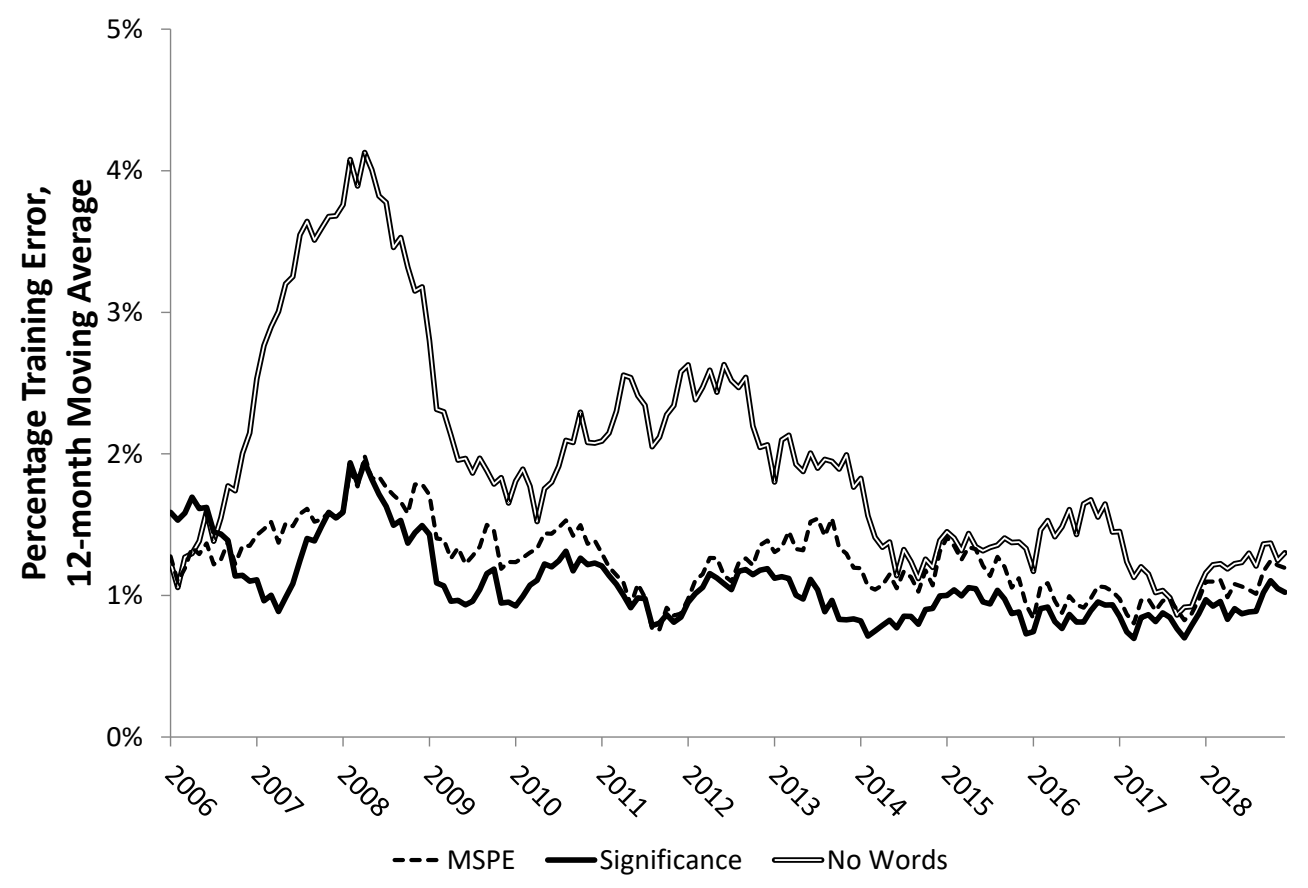


Figure 5: Test Error

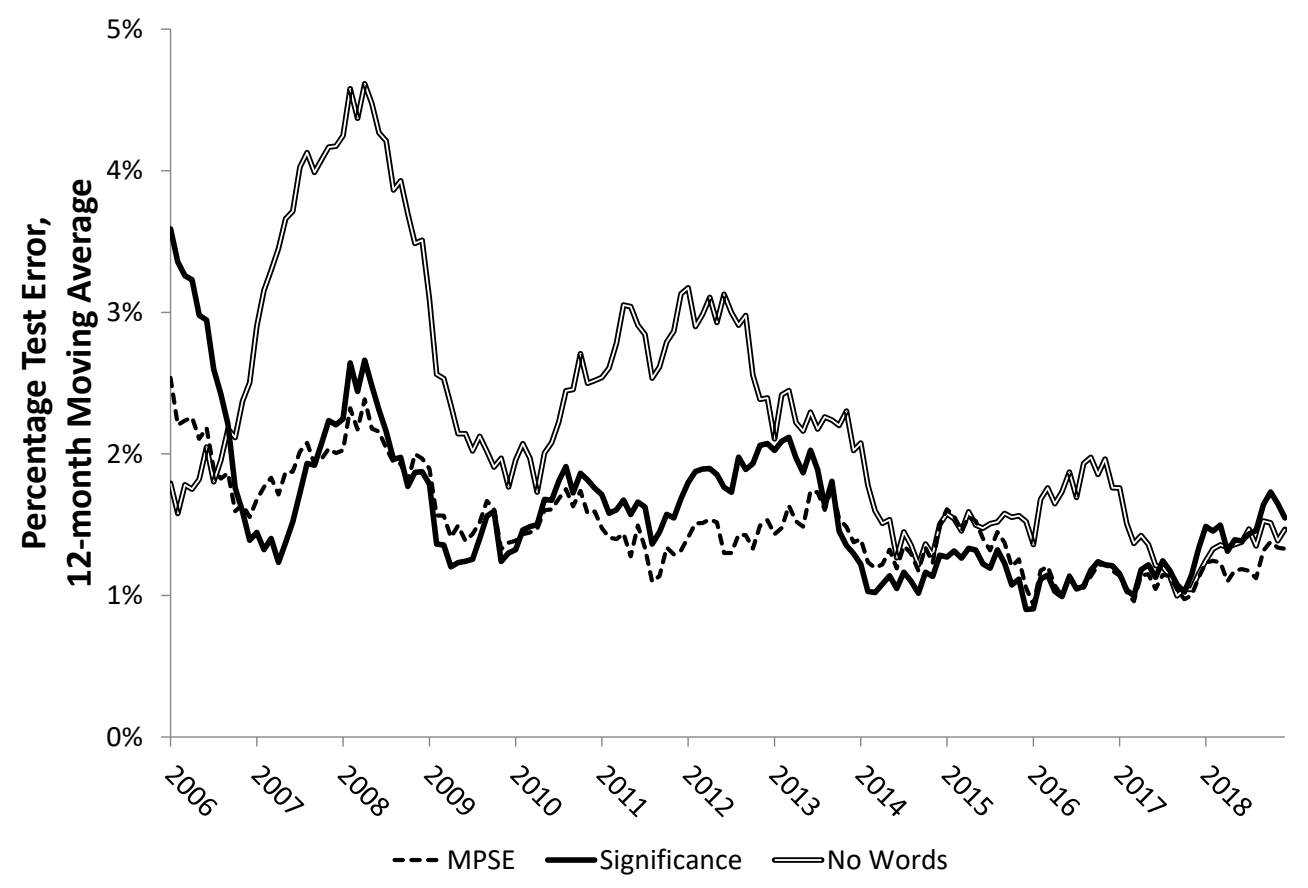


Figure 6: Seasonality-Adjusted Keyword Search Volumes

Clearblue

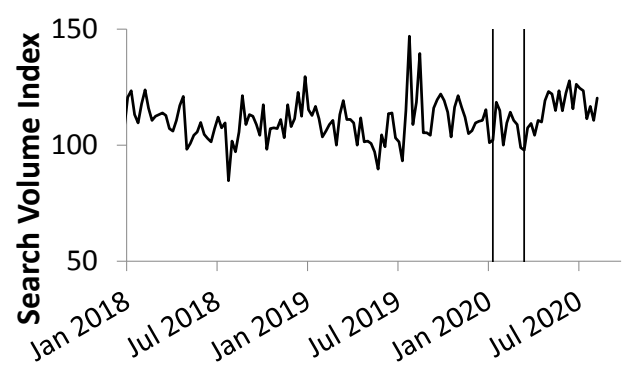

IVF

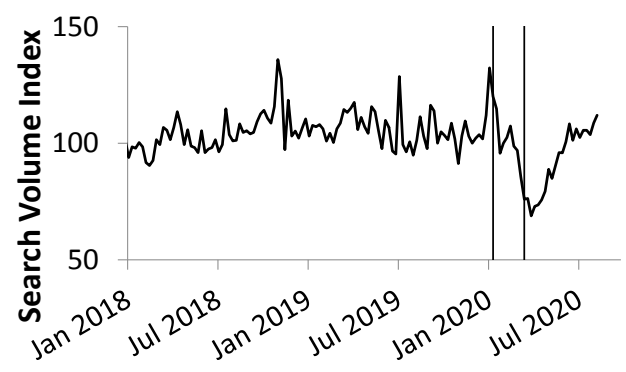

Pregnancy

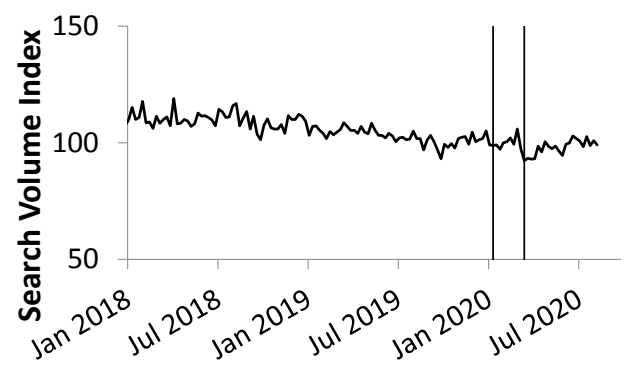

Unemployment

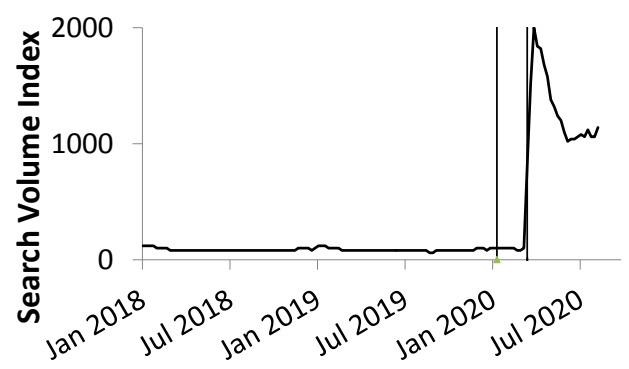

Morning Sickness

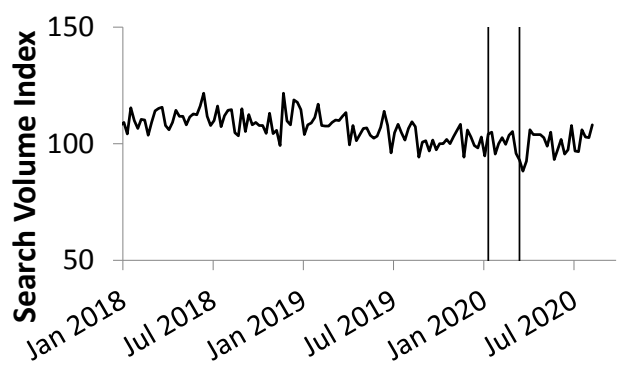

Ovulation

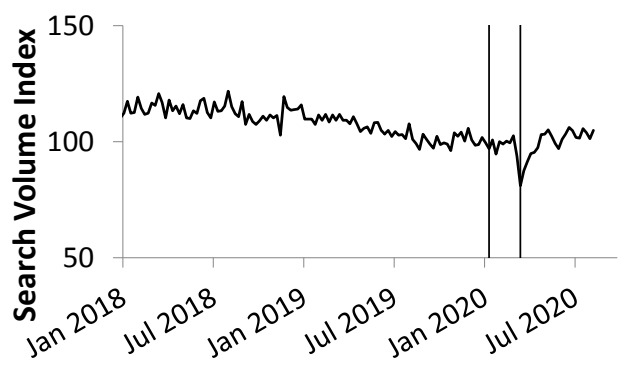

Ultrasound

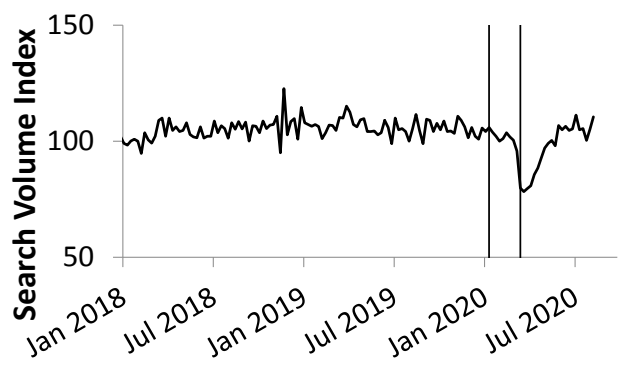

Control Words

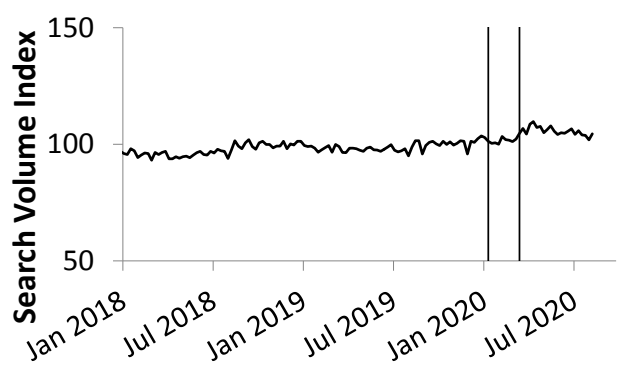

31 
Figure 7: Predicted 2020 US Births by Month

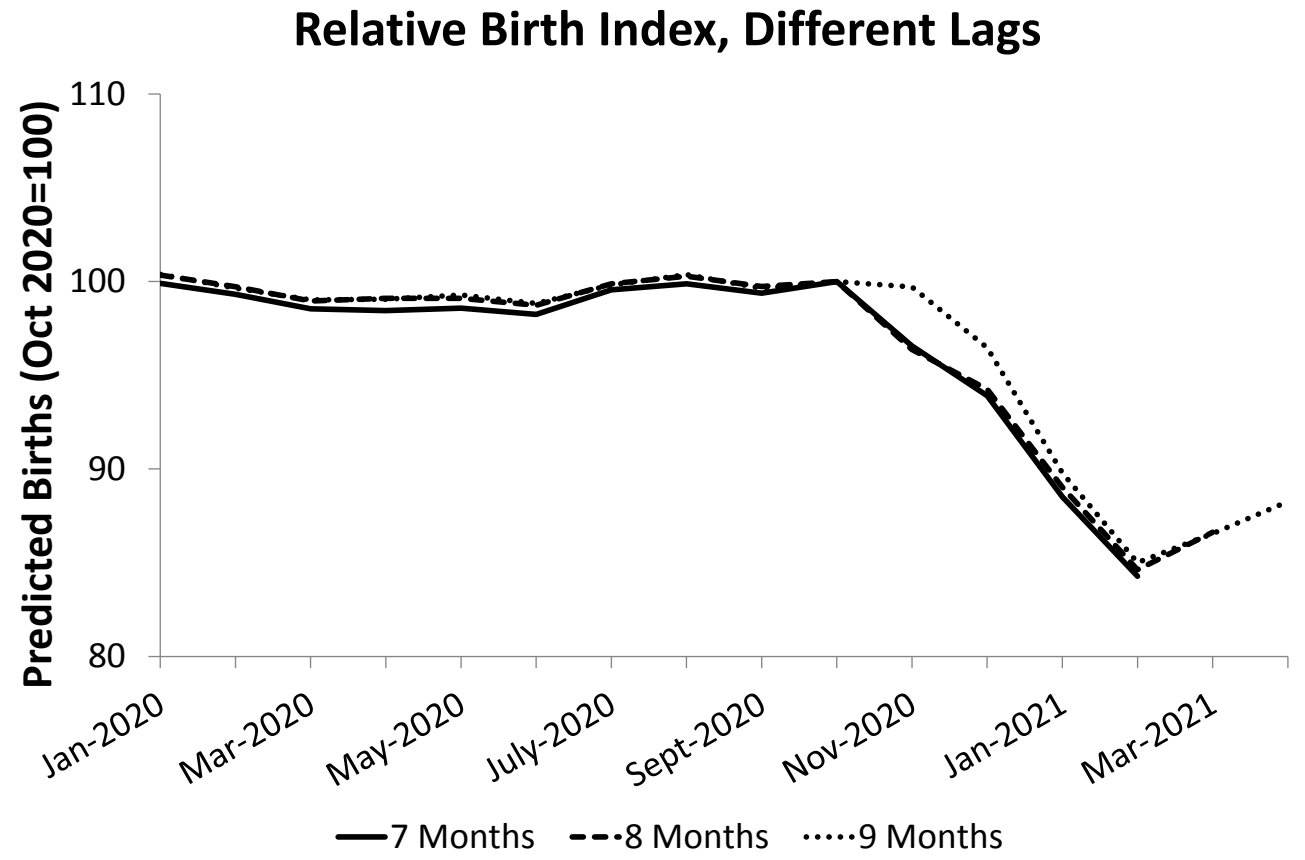

Figure 8: Predicted Fertility Decline by Various Mother Characteristics.
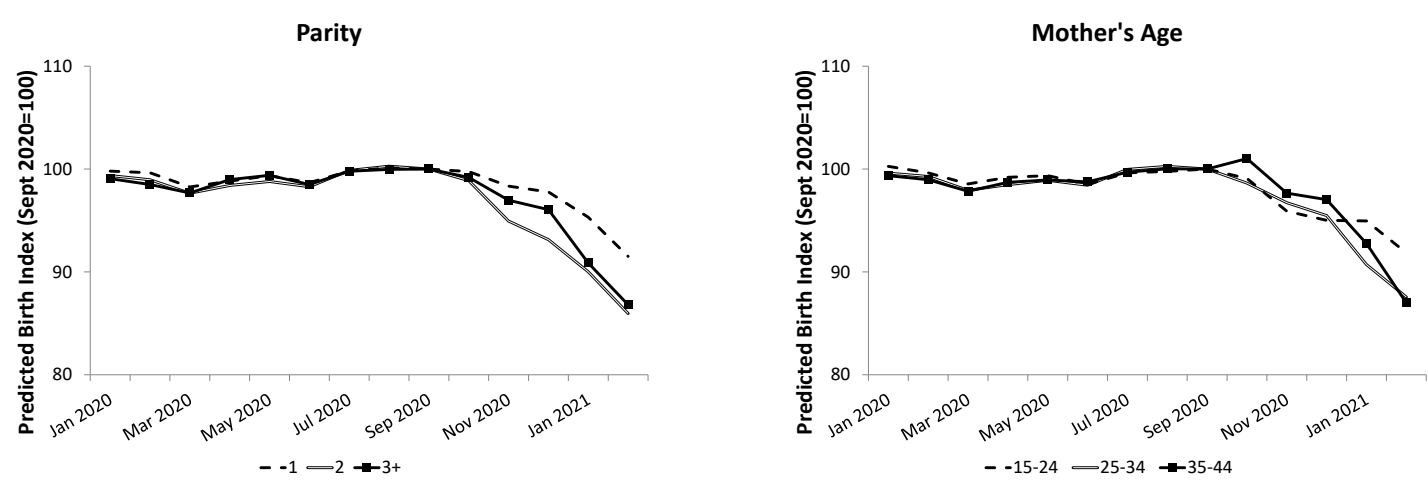
Figure 9: Predicted Fertility Decline between Oct. 2020 and Feb 2021, by State.

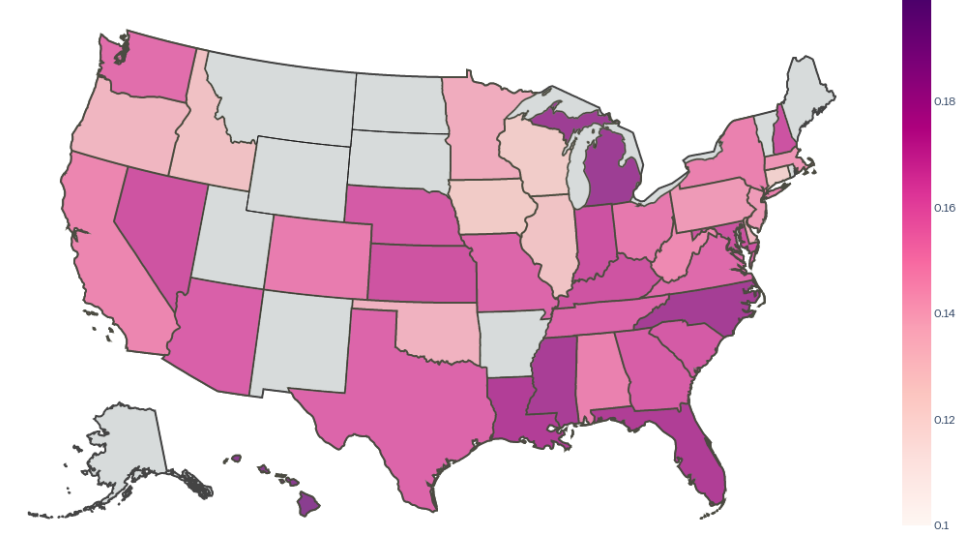

Figure 10: Predicted Fertility Decline and Covid-19 Caseload

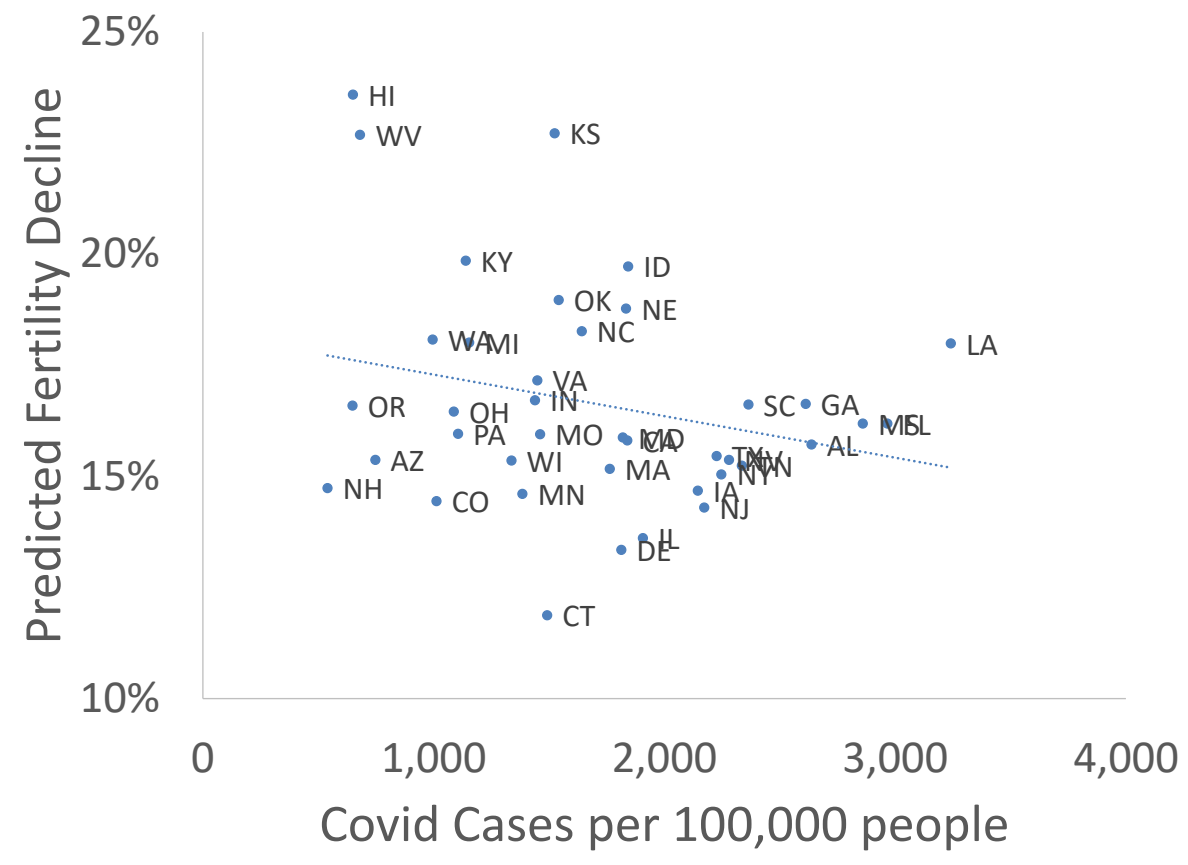


Table 5: Estimated Coefficients Used in Prediction

MSPE Screen Coefficients

\begin{tabular}{lclclcl}
\hline \multicolumn{3}{c}{ Unemployment } & \multicolumn{3}{c}{ Clearblue } \\
\hline $\mathrm{t}-7$ & 0.0010 & $(0.0035)$ & 0.0015 & $(0.0023)$ & $0.0040^{* * *}$ & $(0.0014)$ \\
$\mathrm{t}-8$ & $-0.0134 * * *$ & $(0.0042)$ & $-0.0038^{*}$ & $(0.0022)$ & $0.0058^{* * *}$ & $(0.0014)$ \\
$\mathrm{t}-9$ & -0.0055 & $0.0041)$ & -0.0026 & $(0.0022)$ & 0.0013 & $(0.0014)$ \\
$\mathrm{t}-10$ & $-0.0208^{* * *}$ & $(0.0041)$ & $-0.0078 * * *$ & $(0.0022)$ & 0.0021 & $(0.0014)$ \\
$\mathrm{t}-11$ & $-0.0130^{* * *}$ & $(0.0040)$ & $-0.0078^{* * *}$ & $(0.0022)$ & $0.0031^{* *}$ & $(0.0014)$ \\
$\mathrm{t}-12$ & $0.0120 * * *$ & $(0.0035)$ & $-0.0058^{* * *}$ & $(0.0021)$ & 0.0018 & $(0.0014)$ \\
\hline & Unemp. Office & & Ovulation & & Control & \\
$\mathrm{t}-7$ & 0.0015 & $(0.0018)$ & $0.0126 * * *$ & $(0.0044)$ & $0.0472 * *$ & $(0.0198)$ \\
$\mathrm{t}-8$ & -0.0021 & $(0.0018)$ & $0.0227 * * *$ & $(0.0046)$ & $-0.0373 *$ & $(0.0217)$ \\
$\mathrm{t}-9$ & -0.0014 & $(0.0018)$ & $0.0089 * *$ & $(0.0043)$ & -0.0320 & $(0.0206)$ \\
$\mathrm{t}-10$ & $-0.0040 * *$ & $(0.0018)$ & $0.0082^{*}$ & $(0.0043)$ & 0.0117 & $(0.0210)$ \\
$\mathrm{t}-11$ & -0.0027 & $(0.0018)$ & $-0.0095^{* *}$ & $(0.0041)$ & -0.0212 & $(0.0195)$ \\
$\mathrm{t}-12$ & 0.0025 & $(0.0017)$ & $-0.0105 * *$ & $(0.0043)$ & 0.0219 & $(0.0165)$
\end{tabular}

Significance Screen Coefficients

\begin{tabular}{|c|c|c|c|c|c|c|c|c|}
\hline & Clearblue & & Divorce & & $\mathrm{HCG}$ & & IVF & \\
\hline$t-7$ & $0.0041^{* * * *}$ & $(0.0014)$ & $0.0085^{*}$ & $(0.0046)$ & 0.0011 & $(0.0022)$ & -0.0010 & $(0.0022)$ \\
\hline $\mathrm{t}-8$ & $0.0047 * * *$ & $(0.0014)$ & $0.0117 * * *$ & $(0.0045)$ & $-0.0036^{*}$ & $(0.0021)$ & -0.0002 & $(0.0021)$ \\
\hline $\mathrm{t}-9$ & 0.0012 & $(0.0014)$ & $0.0196^{* * *}$ & $(0.0046)$ & $-0.0042 * *$ & $(0.0021)$ & $-0.0053^{* *}$ & $(0.0023)$ \\
\hline $\mathrm{t}-10$ & 0.0010 & $(0.0013)$ & $0.0101 * *$ & $(0.0044)$ & $-0.0077 * * *$ & $(0.0022)$ & $-0.0079 * * *$ & $(0.0022)$ \\
\hline $\mathrm{t}-11$ & $0.0023^{*}$ & $(0.0014)$ & 0.0037 & $(0.0048)$ & $-0.0066 * * *$ & $(0.0021)$ & -0.0020 & $(0.0022)$ \\
\hline \multirow[t]{2}{*}{$\mathrm{t}-12$} & 0.0004 & $(0.0014)$ & $0.0123 * * *$ & $(0.0044)$ & $-0.0071 * * *$ & $(0.0021)$ & 0.0003 & $(0.0020)$ \\
\hline & Layoff & & Morning Sickness & & Ovulation & & Porn & \\
\hline$t-7$ & 0.0002 & $(0.0015)$ & $0.0061 * * *$ & $(0.0020)$ & $0.0137 * * *$ & $(0.0046)$ & -0.0161 & $(0.0103)$ \\
\hline $\mathrm{t}-8$ & 0.0005 & $(0.0016)$ & $0.0052 * * *$ & $(0.0020)$ & $0.0206^{* * *}$ & $(0.0048)$ & 0.0142 & $(0.0119)$ \\
\hline $\mathrm{t}-9$ & 0.0023 & $(0.0016)$ & 0.0017 & $(0.0020)$ & $0.0087 *$ & $(0.0045)$ & -0.0026 & $(0.0122)$ \\
\hline $\mathrm{t}-10$ & $-0.0061 * * *$ & $(0.0015)$ & 0.0012 & $(0.0020)$ & $0.0107 * *$ & $(0.0045)$ & $0.0361 * * *$ & $(0.0117)$ \\
\hline $\mathrm{t}-11$ & $0.0056^{* * * *}$ & $(0.0015)$ & 0.0004 & $(0.0019)$ & -0.0018 & $(0.0043)$ & 0.0191 & $(0.0124)$ \\
\hline \multirow[t]{2}{*}{$\mathrm{t}-12$} & $-0.0040 * * *$ & $(0.0014)$ & -0.0010 & $(0.0019)$ & -0.0052 & $(0.0043)$ & -0.0088 & $(0.0106)$ \\
\hline & Preg. Test & & Pregnancy & & Pregnant & & STI & \\
\hline$t-7$ & 0.0040 & $(0.0039)$ & $0.0317 * * *$ & $(0.0120)$ & -0.0077 & $(0.0081)$ & -0.0064 & $(0.0041)$ \\
\hline $\mathrm{t}-8$ & $0.0123 * * *$ & $(0.0037)$ & -0.0022 & $(0.0121)$ & -0.0040 & $(0.0080)$ & $-0.0077 *$ & $(0.0041)$ \\
\hline$t-9$ & 0.0046 & $(0.0038)$ & -0.0120 & $(0.0118)$ & -0.0037 & $(0.0077)$ & -0.0025 & $(0.0041)$ \\
\hline $\mathrm{t}-10$ & 0.0059 & $(0.0038)$ & $-0.0468 * * *$ & $(0.0119)$ & -0.0037 & $(0.0075)$ & -0.0027 & $(0.0041)$ \\
\hline $\mathrm{t}-11$ & 0.0065 & $(0.0041)$ & $-0.0326 * * *$ & $(0.0123)$ & $-0.0247 * * *$ & $(0.0078)$ & 0.0036 & $(0.0043)$ \\
\hline \multirow[t]{2}{*}{$\mathrm{t}-12$} & $0.0086^{* *}$ & $(0.0037)$ & -0.0164 & $(0.0118)$ & 0.0079 & $(0.0077)$ & -0.0001 & $(0.0037)$ \\
\hline & Ultrasound & & Unemployment & & Control & & & \\
\hline$t-7$ & 0.0057 & $(0.0038)$ & 0.0033 & $(0.0034)$ & 0.0240 & $(0.0203)$ & & \\
\hline $\mathrm{t}-8$ & $-0.0063^{*}$ & $(0.0037)$ & $-0.0150 * * *$ & $(0.0041)$ & $-0.0438 * *$ & $(0.0221)$ & & \\
\hline $\mathrm{t}-9$ & $-0.0083^{* *}$ & $(0.0036)$ & -0.0054 & $(0.0039)$ & $-0.0377^{*}$ & $(0.0216)$ & & \\
\hline $\mathrm{t}-10$ & $-0.0132 * * *$ & $(0.0040)$ & $-0.0213 * * *$ & $(0.0040)$ & -0.0015 & $(0.0208)$ & & \\
\hline $\mathrm{t}-11$ & $-0.0068 *$ & $(0.0035)$ & $-0.0124 * * *$ & $(0.0040)$ & $-0.0426^{* *}$ & $(0.0206)$ & & \\
\hline $\mathrm{t}-12$ & $-0.0113 * * *$ & $(0.0036)$ & $0.0103 * * *$ & $(0.0036)$ & 0.0232 & $(0.0184)$ & & \\
\hline
\end{tabular}

Huber-White Standard errors in parentheses to the right of each estimate. $* * * 1 \%, * * 5 \%, * 10 \%$ significance levels. 